\title{
Pitch Dynamics of Unmanned Aerial Vehicles
}

\author{
W. F. Phillips ${ }^{1}$ and D. F. Hunsaker ${ }^{2}$ \\ Utah State University, Logan, Utah 84322-4130 \\ N. R. Alley ${ }^{3}$ \\ AeroMorphology LLC, Canton, Georgia 30115 \\ and \\ R. J. Niewoehner ${ }^{4}$ \\ United States Naval Academy, Annapolis, Maryland 21402-5025
}

\begin{abstract}
Dynamic stability requirements for manned aircraft have been in place for many years. However, we cannot expect stability constraints for UAVs to match those for manned aircraft; and dynamic stability requirements specific to UAVs have not been developed. The boundaries of controllability for both remotely-piloted and auto-piloted aircraft must be established before UAV technology can reach its full potential. The development of dynamic stability requirements specific to UAVs could improve flying qualities and facilitate more efficient UAV designs to meet specific mission requirements. As a first step to developing UAV stability requirements in general, test techniques must be established that will allow the stability characteristics of current UAVs to be quantified. This paper consolidates analytical details associated with procedures that could be used to experimentally determine the pitch stability boundaries for good UAV flying qualities. The procedures require determining only the maneuver margin and pitch radius of gyration and are simple enough to be used in an educational setting where resources are limited. The premise is that these procedures could be applied to UAVs now in use, in order to characterize the longitudinal flying qualities of current aircraft. This is but a stepping stone to the evaluation of candidate metrics for establishing flying-quality constraints for unmanned aircraft.
\end{abstract}

\section{Nomenclature}

$\bar{a} \quad=$ axial distance aft from some arbitrary reference point to the center of gravity

$a_{m p} \quad=$ axial distance aft from some arbitrary reference point to the stick-fixed maneuver point

$a_{n p} \quad=$ axial distance aft from some arbitrary reference point to the stick-fixed neutral point

$a_{\text {wing }}=$ axial distance aft from some arbitrary reference point to the wing quarter chord

$C_{m_{n p} 0}=$ traditional neutral-point moment coefficient with $q=\dot{\alpha}=\delta_{e}=0$

$C_{m_{n p}, \bar{q}}=$ change in traditional neutral-point moment coefficient with traditional dimensionless pitch rate

$C_{m_{n p}, \breve{q}}=$ change in traditional neutral-point moment coefficient with dynamic pitch rate

$C_{m_{n p}, \delta_{e}} \quad=$ change in traditional neutral-point moment coefficient with elevator deflection

$C_{W} \quad=$ weight coefficient

$C_{1}, C_{2}, C_{3}=$ vertical components of string tension per unit weight for a trifilar pendulum

$c_{\text {ref }}=$ arbitrary reference chord length

$c_{1}, c_{2}, c_{3}=$ horizontal chord-length projections for a trifilar pendulum

\footnotetext{
${ }^{1}$ Professor, Mechanical and Aerospace Engineering Department, 4130 Old Main Hill. Senior Member AIAA.

${ }^{2} \mathrm{PhD}$ Candidate, Mechanical and Aerospace Engineering Department, 4130 Old Main Hill. Student Member AIAA.

${ }^{3}$ Senior Aerodynamicist, 349 Spring Hill Drive. Member AIAA.

${ }^{4}$ Captain, US Navy, Aerospace Engineering Department, 590 Holloway Road. Member AIAA.
} 


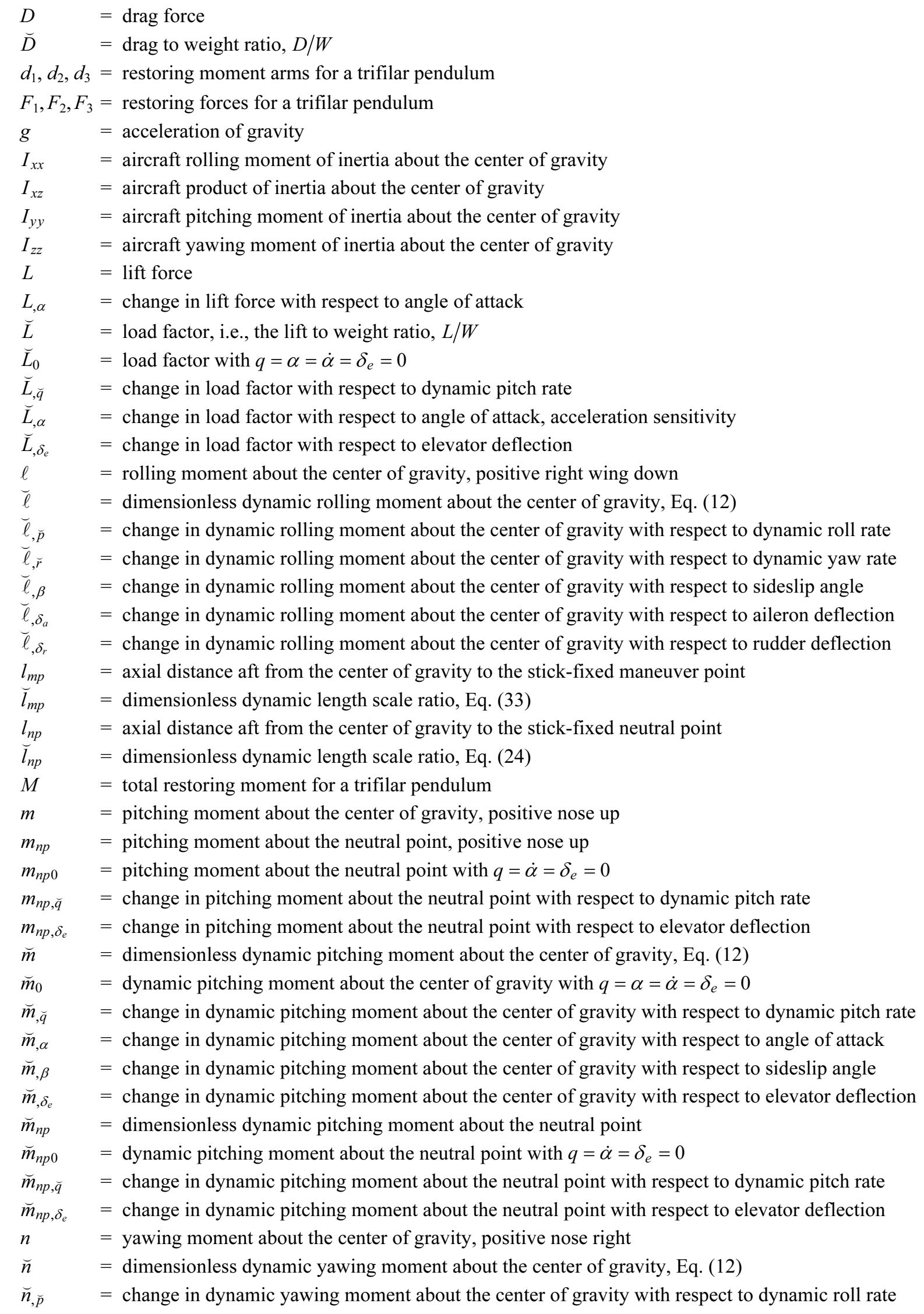




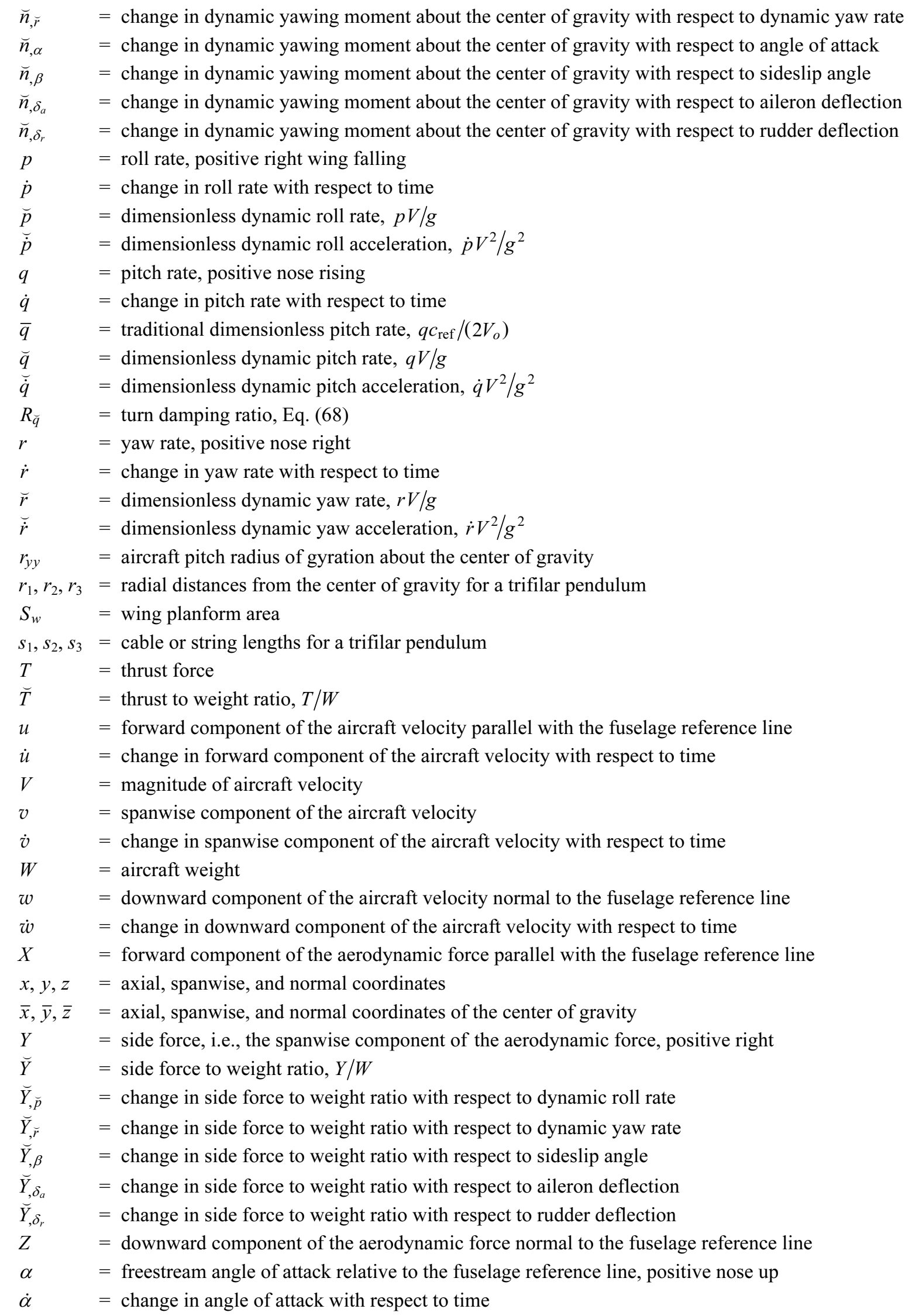




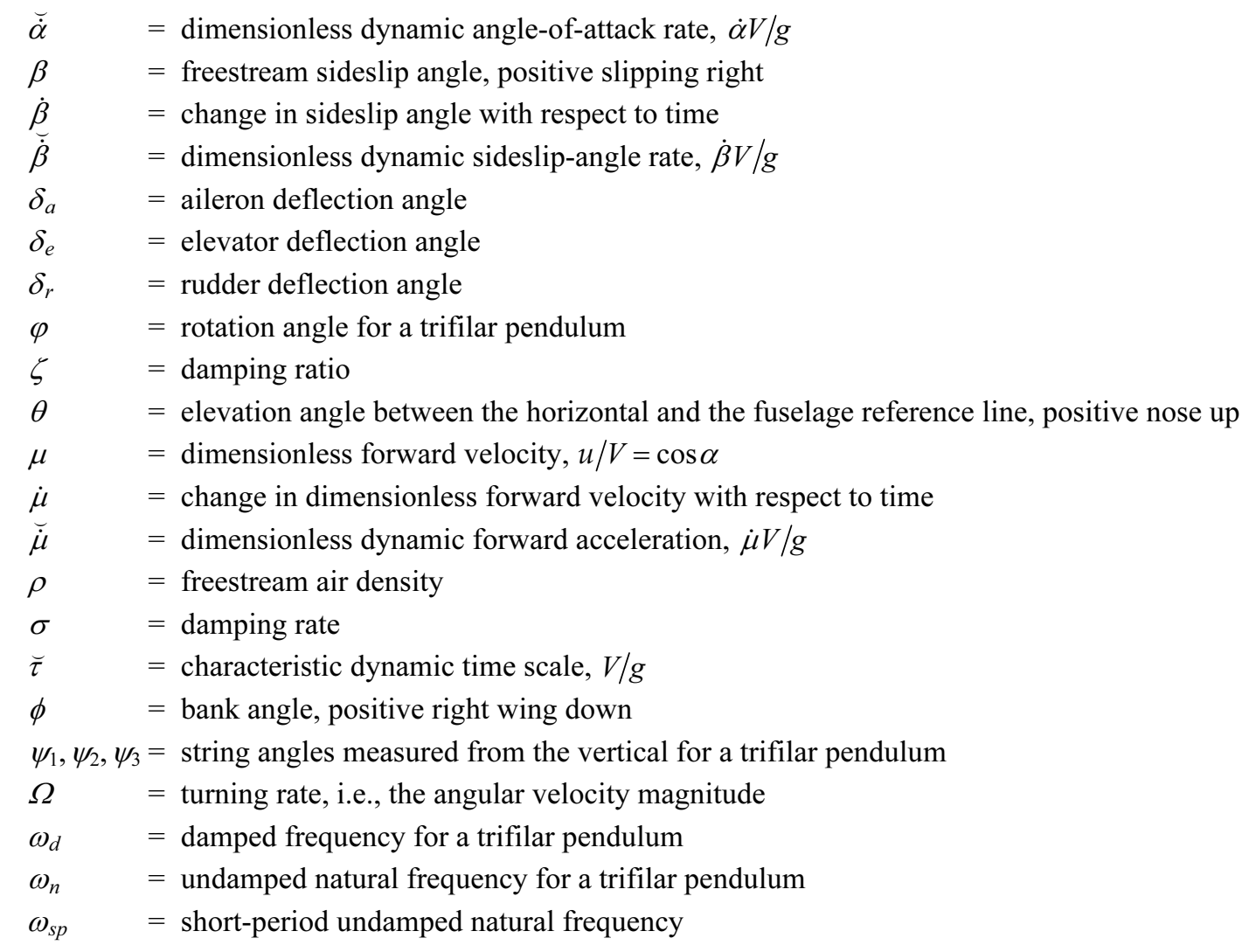

\section{Introduction}

Whereas the Federal Aviation Administration classifies unmanned aerial vehicles (UAVs) based on how they

are used, for our present purpose it makes more sense to classify them based on how they are piloted. Here we will distinguish only two types of UAVs, remotely-piloted aircraft and auto-piloted aircraft.

A remotely-piloted aircraft is an aircraft piloted by a human who is not onboard the aircraft. Remotely-piloted aircraft are commonly referred to as radio control (RC) aircraft. The pilot of an RC aircraft typically evaluates the state of the aircraft solely from ground observations. However, the aircraft may have an embedded camera, which transmits real-time images of the aircraft's surroundings to assist the pilot.

An auto-piloted aircraft is an aircraft piloted by a computer. The computer may be stationed onboard the aircraft or on the ground. The computer obtains information about the state of the aircraft from sensors, transmitters, and receivers, which may be aircraft-based, satellite-based, and/or ground-based.

The use of UAVs has a significant positive impact on Aerospace Engineering Education. For many of us, as children or young adults, our first exposure to the science of human flight was through the recreational/sport use of model aircraft. In the university environment, many engineering students get their first exposure to aircraft design through noncommercial activities associated with designing, building and flying model airplanes. An example of such activities involving remotely-piloted aircraft is the Cessna/Raytheon/AIAA Student Design/Build/Fly competition (http://www.aiaadbf.org/). An example involving auto-piloted aircraft is the Association for Unmanned Vehicle Systems International Student Unmanned Air Systems Competition (http://www.auvsi.org/competitions/).

One problem associated with the design and safe operation of UAVs, whether remotely-piloted or auto-piloted, is the lack of data on dynamic stability requirements for UAVs. For manned airplanes, the publication of stability requirements allows designers to approach flight testing with confidence that the aircraft has been adequately designed for good handling. Although there is a large volume of legacy data that has been used to define standards with respect to dynamic stability requirements for manned aircraft, ${ }^{3,4}$ similar requirements are not available for UAVs. For lack of an alternative, UAV designers commonly use stability requirements developed for manned aircraft. This carries the risk of either over-designing or under-designing a UAV that need not be constrained by the limits of human physiology. Manned aircraft stability requirements were defined through thousands of hours of flight testing, and considerable work is needed to determine the stability requirements for UAVs. As a preface, 
current UAVs, whether remotely-piloted or auto-piloted, need to be documented and characterized in order to begin to understand the characteristics of these aircraft. The development of improved stability requirements specific to UAVs could contribute significantly to the safe and efficient operation of UAVs in all applications.

The dynamic characteristics of an aircraft are often rated in terms of what are commonly called flying qualities or handling qualities. In order to ensure that pilots can maneuver an aircraft to accomplish specific mission requirements, the dynamic characteristics of the airplane should fall within specific limits. Extensive research ${ }^{3,4}$ has shown that manned aircraft flying qualities are related to how well the dynamic modes of the aircraft fit within constraints imposed by pilot limitations and mission requirements. A significant parameter associated with longitudinal flying qualities is the control anticipation parameter (CAP), which is defined to be the ratio of the square of the short-period undamped natural frequency to what is commonly known as the acceleration sensitivity, $\mathrm{CAP} \equiv \omega_{s p}^{2} /\left(L_{, \alpha} / W\right)$ (Ref. 4).

Through thousands of hours of flight testing, correlations have been found between the CAP and pilot opinions of the flying qualities of conventional manned airplanes. These data, which are reported in the United States military specifications MIL-F-8785C ${ }^{3}$ and MIL-STD-1797A, ${ }^{4}$ were used to define constraints on the CAP that are required to ensure acceptable flying qualities for conventional manned airplanes. The CAP constraints for manned airplanes can be expressed as a function of defined flying-quality levels and flight-phase categories ${ }^{3,4}$

$$
\left\{\begin{array}{l}
\left(\begin{array}{l}
0.28 \mathrm{~s}^{-2}, \text { Level } 1 \\
0.15 \mathrm{~s}^{-2}, \text { Level } 2
\end{array}\right), \text { Category A } \\
\left(\begin{array}{l}
0.085 \mathrm{~s}^{-2}, \text { Level } 1 \\
0.038 \mathrm{~s}^{-2}, \text { Level 2 }
\end{array}\right), \text { Category B } \\
\left(\begin{array}{l}
0.15 \mathrm{~s}^{-2}, \text { Level 1 } \\
0.096 \mathrm{~s}^{-2}, \text { Level 2 }
\end{array}\right), \text { Category C }
\end{array}\right\} \leq \frac{\omega_{s p}^{2}}{L_{, \alpha} / W} \leq\left\{\left(\begin{array}{l}
3.6 \mathrm{~s}^{-2}, \text { Level 1 } \\
10 . \mathrm{s}^{-2}, \text { Level 2 }
\end{array}\right), \text { All Categories }\right\}
$$

For the reader who may not be familiar with the military CAP requirements given by Eq. (1), the definitions for flying-quality levels and flight-phase categories that are used in the military specifications are also summarized by Hodgkinson. ${ }^{5}$ The Level-1, Category-A limit should be applied to demanding flight tasks such as air-to-air combat, aerobatics, and close-formation flying, which require rapid maneuvering and precise control. The Level-1, Category-B limit is used for cruise, climb, and other flight phases that are normally accomplished with gradual maneuvers without precision tracking. The Level-1, Category-C limit applies to takeoff, landing, and other flight phases that require accurate flight-path control with gradual maneuvering. The Level-2 limits are usually considered to be acceptable only in a failure state.

The minimum CAP constraint given in Eq. (1) is an experimentally-evaluated function of the flight-phase requirements. This constraint is thought to vary with the mission task at hand partly because of the human pilot's sensitivity to aircraft acceleration. When an aircraft is remotely piloted, the pilot's total physiological sensitivity to aircraft acceleration is replaced with only a visual interpretation of the aircraft acceleration. Furthermore, when an autopilot is used, the reaction of the autopilot to acceleration is based solely on instrumentation and electronic response time. Thus, just as the minimum CAP constraint given in Eq. (1) varies with flight phase for manned aircraft, one should expect this constraint to differ for UAVs whether remotely piloted or auto piloted.

Preliminary flight-test data presented by Foster and Bowman ${ }^{6,7}$ indicate that pilot opinions of the flying qualities of remotely-piloted UAVs do not match the requirements given by Eq. (1). Their preliminary findings suggest that minimum CAP constraints for remotely-piloted UAVs lie well above those for conventional manned aircraft. Thus, it appears that manned aircraft specifications applied to remotely-piloted UAVs are not conservative with respect to safety. This is not surprising when one considers the reduced sensory feedback available to the pilot of an RC aircraft compared with that available to the pilot of a manned aircraft. It is possible that CAP constraints could be developed for remotely-piloted UAVs just as for manned aircraft. However, sufficient flight-test data are not publicly available to define such constraints.

Similar CAP constraints could possibly be determined for auto-piloted UAVs. Because of an autopilot's short response time and precise sensitivity to accelerations, it is likely that autopilot CAP constraints lie well outside the constraints for manned aircraft. Indeed, autopilots seem to have little trouble accurately piloting aircraft that fit within the manned-aircraft CAP constraints. Thus, it appears that applying manned aircraft specifications to auto- 
piloted UAVs is conservative and provides the desired safety, but this may come at the cost of reduced performance. This could be of critical importance in view of the extraordinary performance that is now being asked from some UAVs. It is possible that improved dynamic stability requirements for auto-piloted UAVs could significantly increase performance. However, as with remotely-piloted UAVs, sufficient flight-test data are not publicly available to define the flying-quality constraints for auto-piloted UAVs.

Today, nearly all UAVs are flown, at least at times during the development phase, as remotely-piloted aircraft. A common procedure in UAV development is to have a person on the ground pilot the UAV during takeoff and landing. At least in the early phases of development, the autopilot is given control of the UAV only at a safe distance from the ground. Thus, the stability requirements of remotely-piloted airplanes are and will continue to be important as the technology of UAV flight continues to evolve. As a first step to developing stability requirements for UAVs in general, test techniques must be established, which allow the stability characteristics of remotelypiloted airplanes to be quantified.

Methods for identifying the stability and control characteristics of aircraft have been in place for years. For example, Norton ${ }^{8,9}$ measured longitudinal and lateral frequencies of an aircraft using flight test data in 1923. For a detailed discussion of system identification methods and their application to aircraft in the time domain and frequency domain, the reader is referred to Tischler and Remple, ${ }^{10}$ Jategaonkar, ${ }^{11}$ and Klein and Morelli. ${ }^{12}$ A concise survey of the literature on aircraft system identification methods up to 1980 is given by Iliff. ${ }^{13}$ The purpose here is not to provide a detailed history or extensive overview of aircraft system identification. Rather, it is to set the present paper into perspective relative to prior work.

In its general sense, system identification is a method of estimating the identifying characteristics and parameters of a system based on measured inputs and outputs. Techniques for estimating the error associated with the measurements as well as estimating the best parameters for aircraft have been studied in detail. ${ }^{14-16}$ These techniques have been applied to offline ${ }^{17}$ and online ${ }^{18,19}$ aircraft system identification and have also been used to determine the aeroelastic properties of aircraft. ${ }^{20-22}$

Parameter estimation is a subset of system identification in which the governing equations of the system are assumed to be known and the parameters of the equations, which best model the system, need to be determined. Errors resulting from noise in the input and output measurements, as well as errors associated with the model, require some form of estimation to obtain the best parameters for a system in the presence of noise. The best set of parameters is the set that minimizes the difference between the model and measured output of the system. Thus, the goal is to find those parameters that allow the model to best match the actual dynamics of the system, rather than trying to determine the actual parameters of the system. Methods for estimating these parameters can be found in many system identification books and include the least-squares estimate,,$^{23,24}$ and maximum likelihood estimate. ${ }^{25,26}$

As stated previously, a significant parameter in longitudinal stability and control is the CAP. The CAP has traditionally been estimated by measuring the short-period natural frequency and acceleration sensitivity of an aircraft using system identification methods. This paper presents an alternative procedure through which the CAP can be experimentally determined without exciting the short-period natural frequency. The method and required tools are simple enough to be implemented on a remotely-piloted aircraft in an educational setting where resources for extensive flight testing are often limited.

The CAP is currently defined as the square of the short-period natural frequency divided by the acceleration sensitivity. It has been shown that within the assumptions of linear aerodynamics and small disturbances, the CAP can be written in terms of the maneuver margin, the pitch radius of gyration, and the acceleration of gravity, ${ }^{27}$

$$
\mathrm{CAP} \equiv \frac{\omega_{s p}^{2}}{L_{, \alpha} / W}=\frac{l_{m p} g}{r_{y y}^{2}}
$$

Therefore, the CAP for an aircraft can be accurately determined by measuring only two parameters of the aircraft; the maneuver margin and the pitch radius of gyration.

Developing realistic CAP constraints for remotely-piloted aircraft will require the correlation of data from extensive flight testing by many pilots. These flight tests will likely involve shifting the center of gravity (CG) until the aircraft reaches points of degraded controllability. ${ }^{6,7}$ The shift in CG affects both the maneuver margin and the pitch radius of gyration. This paper consolidates the details of one established method for experimentally determining each of these two parameters, and the methodology presented accounts for the effects of shifting the CG location. The premise is that the methods included in this paper could be applied to remotely-piloted aircraft now in 
use, in order to characterize the longitudinal flying qualities of current aircraft. This is but a stepping stone to the evaluation of candidate metrics for establishing flying-quality constraints for remotely-piloted aircraft.

\section{Steady Linearized Dynamics}

The distribution of longitudinal aerodynamic loads acting on an airplane can be replaced with an axial force, $X$, a normal force, $Z$, and a pitching moment, $m$, acting at the center of gravity (CG). Similarly, the distribution of lateral aerodynamic loads can be resolved into a side force, $Y$, a rolling moment, $\ell$, and a yawing moment, $n$, also acting at the CG. Because the orientation of the fuselage reference line is arbitrary, here it is defined to be aligned with the thrust vector. Thus, neglecting the nonlinear effects of vertical offsets, Newton's second law and the angular momentum equation about the CG can be written as ${ }^{28-32}$

$$
\left\{\begin{array}{c}
(W / g)(\dot{u}+w q-v r) \\
(W / g)(\dot{v}+u r-w p) \\
(W / g)(\dot{w}+v p-u q) \\
I_{x x} \dot{p}+\left(I_{z z}-I_{y y}\right) q r-I_{x z}(p q+\dot{r}) \\
I_{y y} \dot{q}+\left(I_{x x}-I_{z z}\right) p r+I_{x z}\left(p^{2}-r^{2}\right) \\
I_{z z} \dot{r}+\left(I_{y y}-I_{x x}\right) p q+I_{x z}(q r-\dot{p})
\end{array}\right\}=\left\{\begin{array}{c}
T+X-W \sin \theta \\
Y+W \cos \theta \sin \phi \\
Z+W \cos \theta \cos \phi \\
\ell \\
m \\
n
\end{array}\right\}
$$

The axial and normal force components can be expressed in terms of lift and drag,

$$
\left\{\begin{array}{l}
X \\
Z
\end{array}\right\}=\left[\begin{array}{cc}
\sin \alpha & -\cos \alpha \\
-\cos \alpha & -\sin \alpha
\end{array}\right]\left\{\begin{array}{l}
L \\
D
\end{array}\right\}
$$

At small angles of attack, drag contributes little to the normal force. Thus, applying the small-angle approximations ( $\cos \alpha \cong 1$ and $\sin \alpha \cong \alpha$ ), we neglect drag in the second component of Eq. (4) to obtain

$$
\left\{\begin{array}{l}
X \\
Z
\end{array}\right\} \cong\left\{\begin{array}{c}
L \alpha-D \\
-L
\end{array}\right\}
$$

Assuming small changes in airspeed (i.e., $u \equiv V \mu, v \cong V \beta, w \cong V \alpha, \dot{u} \cong V \dot{\mu}, \dot{v} \cong V \dot{\beta}$, and $\dot{w} \cong V \dot{\alpha}$ ) and using Eq. (5) in Eq. (3), the small-angle equations of motion for maneuvering flight at nearly constant airspeed yield

$$
\left\{\begin{array}{c}
W \dot{\mu} V / g \\
W \dot{\beta} V / g \\
W \dot{\alpha} V / g \\
I_{x x} \dot{p} \\
I_{y y} \dot{q} \\
I_{z z} \dot{r}
\end{array}\right\}=\left\{\begin{array}{c}
T-D+L \alpha-W[\sin \theta+(q \alpha-r \beta) V / g] \\
Y+W[\cos \theta \sin \phi-(r \mu-p \alpha) V / g] \\
-L+W[\cos \theta \cos \phi+(q \mu-p \beta) V / g] \\
\ell+\left(I_{y y}-I_{z z}\right) q r+I_{x z}(\dot{r}+p q) \\
m+\left(I_{z z}-I_{x x}\right) p r+I_{x z}\left(r^{2}-p^{2}\right) \\
n+\left(I_{x x}-I_{y y}\right) p q+I_{x z}(\dot{p}-q r)
\end{array}\right\}
$$

The characteristic time scale that appears naturally in the components of Newton's second law was identified by Phillips and Niewoehner ${ }^{27}$ and referred to as the dynamic time scale,

$$
\breve{\tau} \equiv V / g
$$

Continuing to follow Phillips and Niewoehner, ${ }^{27}$ we define the dimensionless dynamic rates and accelerations

$$
\begin{gathered}
\breve{\dot{\mu}} \equiv \dot{\mu} V / g, \quad \breve{\dot{\beta}} \equiv \dot{\beta} V / g, \breve{\alpha} \equiv \dot{\alpha} V / g, \quad \breve{p} \equiv p V / g, \breve{q} \equiv q V / g, \text { and } \breve{r} \equiv r V / g \\
\breve{\dot{p}} \equiv \dot{p} V^{2} / g^{2}, \breve{\dot{q}} \equiv \dot{q} V^{2} / g^{2}, \text { and } \breve{\dot{r}} \equiv \dot{r} V^{2} / g^{2}
\end{gathered}
$$


Using Eqs. (8) and (9) in Eq. (6) produces the dimensionless system of first-order differential equations

$$
\left\{\begin{array}{c}
\breve{\dot{\mu}} \\
\dot{\dot{\beta}} \\
\check{\dot{\alpha}} \\
\breve{\dot{p}} \\
\breve{\dot{q}} \\
\breve{\dot{r}}
\end{array}\right\}=\left\{\begin{array}{c}
T / W-(D / W)+(L / W) \alpha-\sin \theta-\breve{q} \alpha+\breve{r} \beta \\
Y / W+\cos \theta \sin \phi-\breve{r} \mu+\breve{p} \alpha \\
-L / W+\cos \theta \cos \phi+\breve{q} \mu-\breve{p} \beta \\
\ell V^{2} /\left(g^{2} I_{x x}\right)+\left[\left(I_{y y}-I_{z z}\right) / I_{x x}\right] \breve{q} \breve{r}+\left(I_{x z} / I_{x x}\right)(\breve{\dot{r}}+\breve{p} \breve{q}) \\
m V^{2} /\left(g^{2} I_{y y}\right)+\left[\left(I_{z z}-I_{x x}\right) / I_{y y}\right] \breve{p} \breve{r}+\left(I_{x z} / I_{y y}\right)\left(\breve{r}^{2}-\breve{p}^{2}\right) \\
n V^{2} /\left(g^{2} I_{z z}\right)+\left[\left(I_{x x}-I_{y y}\right) / I_{z z}\right] \breve{p} \breve{q}+\left(I_{x z} / I_{z z}\right)(\dot{p}-\breve{q} \breve{r})
\end{array}\right\}
$$

We see from Eq. (10) that using the characteristic dynamic time scale defined by Eq. (7) to nondimensionalize the rates and accelerations leads naturally to definitions for dimensionless dynamic force and moment components. Here we shall use the notation

$$
\breve{T} \equiv T / W, \breve{D} \equiv D / W, \breve{L} \equiv L / W, \text { and } \breve{Y} \equiv Y / W
$$

and

$$
\breve{\ell} \equiv \ell V^{2} /\left(g^{2} I_{x x}\right), \breve{m} \equiv m V^{2} /\left(g^{2} I_{y y}\right), \text { and } \breve{n} \equiv n V^{2} /\left(g^{2} I_{z z}\right)
$$

The dimensionless dynamic thrust, $\breve{T}$, is simply the commonly-used thrust-to-weight ratio and $\breve{L}$ is the well-known load factor, which is traditionally given the symbol $n$. However, here we will continue to denote the load factor as $\breve{L}$ to avoid confusion with the yawing moment, which is also traditionally given the symbol $n$.

For steady maneuvering flight, airspeed is constant $(\mu=1)$ and the time derivatives in Eq. (10) are zero, so the only acceleration components are the centripetal and Coriolis accelerations. Thus, after using Eqs. (11) and (12) in Eq. (10) and rearranging, steady maneuvering flight requires

$$
\left\{\begin{array}{c}
\breve{T} \\
\breve{L} \\
\breve{Y} \\
\breve{\ell} \\
\breve{m} \\
\breve{n}
\end{array}\right\}=\left\{\begin{array}{c}
\breve{D}+\sin \theta+(\breve{q}-\breve{L}) \alpha-\breve{r} \beta \\
\cos \theta \cos \phi+\breve{q}-\breve{p} \beta \\
-\cos \theta \sin \phi+\breve{r}-\breve{p} \alpha \\
{\left[\left(I_{z z}-I_{y y}\right) / I_{x x}\right] \breve{q} \breve{r}-\left(I_{x z} / I_{x x}\right) \breve{p} \breve{q}} \\
{\left[\left(I_{x x}-I_{z z}\right) / I_{y y}\right] \breve{p} \breve{r}+\left(I_{x z} / I_{y y}\right)\left(\breve{p}^{2}-\breve{r}^{2}\right)} \\
{\left[\left(I_{y y}-I_{x x}\right) / I_{z z}\right] \breve{p} \breve{q}+\left(I_{x z} / I_{z z}\right) \breve{q} \breve{r}}
\end{array}\right\}
$$

When combined with an appropriate aerodynamic model, the first component of Eq. (13) specifies the thrust required to maintain steady flight and the remaining five components determine the two aerodynamic angles and three control surface deflections that are required for a particular steady maneuver.

A fairly general model for the lift, side force, and aerodynamic moments during steady maneuvering flight in the range of linear aerodynamics can be written in terms of the dynamic variables as

$$
\left\{\begin{array}{c}
\breve{L} \\
\breve{Y} \\
\breve{\ell} \\
\breve{m} \\
\breve{n}
\end{array}\right\}=\left\{\begin{array}{c}
\breve{L}_{0} \\
0 \\
0 \\
\breve{m}_{0} \\
0
\end{array}\right\}+\left[\begin{array}{ccccc}
\breve{L}_{, \alpha} & 0 & 0 & \breve{L}_{, \delta_{e}} & 0 \\
0 & \breve{Y}_{, \beta} & \breve{Y}_{\delta_{a}} & 0 & \breve{Y}_{, \delta_{r}} \\
0 & \breve{\ell}_{, \beta} & \bar{\ell}_{, \delta_{a}} & 0 & \bar{\ell}_{, \delta_{r}} \\
\breve{m}_{, \alpha} & \breve{m}_{, \beta} & 0 & \breve{m}_{, \delta_{e}} & 0 \\
\breve{n}_{, \alpha} & \breve{n}_{, \beta} & \breve{n}_{, \delta_{a}} & 0 & \breve{n}_{, \delta_{r}}
\end{array}\right]\left\{\begin{array}{l}
\alpha \\
\beta \\
\delta_{a} \\
\delta_{e} \\
\delta_{r}
\end{array}\right\}+\left[\begin{array}{ccc}
0 & \breve{L}_{\breve{q}} & 0 \\
\breve{Y}_{\breve{p}} & 0 & \breve{Y}_{\breve{r}} \\
\bar{\ell}_{, \breve{p}} & 0 & \breve{\ell}_{\breve{r}} \\
0 & \breve{m}_{, \breve{q}} & 0 \\
\breve{n}_{, \breve{p}} & 0 & \breve{n}_{, \breve{r}}
\end{array}\right]\left\{\begin{array}{l}
\breve{p} \\
\breve{q} \\
\breve{r}
\end{array}\right\}
$$

Note that the pitching moment is taken as a linear function of $\beta$ and the yawing moment is considered to be a linear function of $\alpha$. These longitudinal-lateral coupling terms are included to allow for aerodynamic coupling such as that generated by the propeller of a single-engine airplane. Using Eq. (14) in the last five components of Eq. (13) yields 


$$
\left[\begin{array}{ccccc}
\breve{L}_{, \alpha} & \breve{p} & 0 & \breve{L}_{, \delta_{e}} & 0 \\
\breve{p} & \breve{Y}_{, \beta} & \breve{Y}_{, \delta_{a}} & 0 & \breve{Y}_{, \delta_{r}} \\
0 & \breve{\ell}_{, \beta} & \breve{\ell}_{, \delta_{a}} & 0 & \breve{\ell}_{, \delta_{r}} \\
\breve{m}_{, \alpha} & \breve{m}_{, \beta} & 0 & \breve{m}_{, \delta_{e}} & 0 \\
\breve{n}_{, \alpha} & \breve{n}_{, \beta} & \breve{n}_{, \delta_{a}} & 0 & \breve{n}_{, \delta_{r}}
\end{array}\right]\left\{\begin{array}{c}
\alpha \\
\beta \\
\delta_{a} \\
\delta_{e} \\
\delta_{r}
\end{array}\right\}=\left\{\begin{array}{c}
\cos \theta \cos \phi-\breve{L}_{0}+\left(1-\breve{L}_{, \breve{q}}\right) \breve{q} \\
-\cos \theta \sin \phi-\breve{Y}_{, \breve{p}} \breve{p}+\left(1-\breve{Y}_{\breve{r}}\right) \breve{r} \\
{\left[\left(I_{z z}-I_{y y}\right) / I_{x x}\right] \breve{q} \breve{r}-\left(I_{x z} / I_{x x}\right) \breve{p} \breve{q}-\breve{\ell}_{, \breve{p}} \breve{p}-\breve{\ell}_{,} \breve{r}} \\
-\breve{m}_{0}+\left[\left(I_{x x}-I_{z z}\right) / I_{y y}\right] \breve{p} \breve{r}+\left(I_{x z} / I_{y y}\right)\left(\breve{p}^{2}-\breve{r}^{2}\right)-\breve{m}_{, \breve{q}} \breve{q} \\
{\left[\left(I_{y y}-I_{x x}\right) / I_{z z}\right] \breve{p} \breve{q}+\left(I_{x z} / I_{z z}\right) \breve{q} \breve{r}-\breve{n}_{, \breve{p}} \breve{p}-\breve{n}_{, \breve{r}} \breve{r}}
\end{array}\right\}
$$

Equation (15) can be used to evaluate the two aerodynamic angles and three control surface deflections for a steady maneuver from specified values of the orientation angles and angular rates.

Equation (15) differs from the traditional dynamic formulation only with respect to the dynamic length and time scales that are used to nondimensionalize the formulation. Flight-test data reported in U.S. military specifications s $^{3,4}$ show that flying qualities of manned airplanes do not scale with traditional nondimensional parameters. ${ }^{27}$ The use of more physically significant dynamic length and time scales will likely prove advantageous as we begin to develop dynamic stability requirements for UAVs, which will span a range of vehicle size several orders of magnitude greater than the extent spanned by manned aircraft.

\section{Elevator Angle per $\mathrm{g}$}

The elevator angle per $g$ is traditionally defined to be the change in elevator deflection with respect to load factor at $\theta=0$ for the quasi-steady pull-up maneuver, which is shown in Fig. 1. Although the time derivatives are seldom precisely zero in a constant-speed pull-up maneuver, the elevator angle per $g$ is traditionally defined in terms of the steady limit. By definition, this is a longitudinal maneuver, so $\phi, p$, and $r$ are also zero. The centripetal acceleration for the quasi-steady pull-up maneuver can be written in terms of the load factor, i.e.,

$$
V q=\frac{L-W \cos \theta}{W / g}=(\breve{L}-\cos \theta) g
$$

Thus, the body-fixed angular velocity components for the quasi-steady pull-up maneuver can be expressed in terms of the load factor,

$$
\left\{\begin{array}{l}
p \\
q \\
r
\end{array}\right\}=\left\{\begin{array}{c}
0 \\
(\breve{L}-\cos \theta) g / V \\
0
\end{array}\right\} \quad \text { or } \quad\left\{\begin{array}{l}
\breve{p} \\
\breve{q} \\
\breve{r}
\end{array}\right\}=\left\{\begin{array}{c}
0 \\
\breve{L}-\cos \theta \\
0
\end{array}\right\}
$$

Applying Eq. (17) to Eq. (15) and setting the elevation angle and bank angle to zero yields

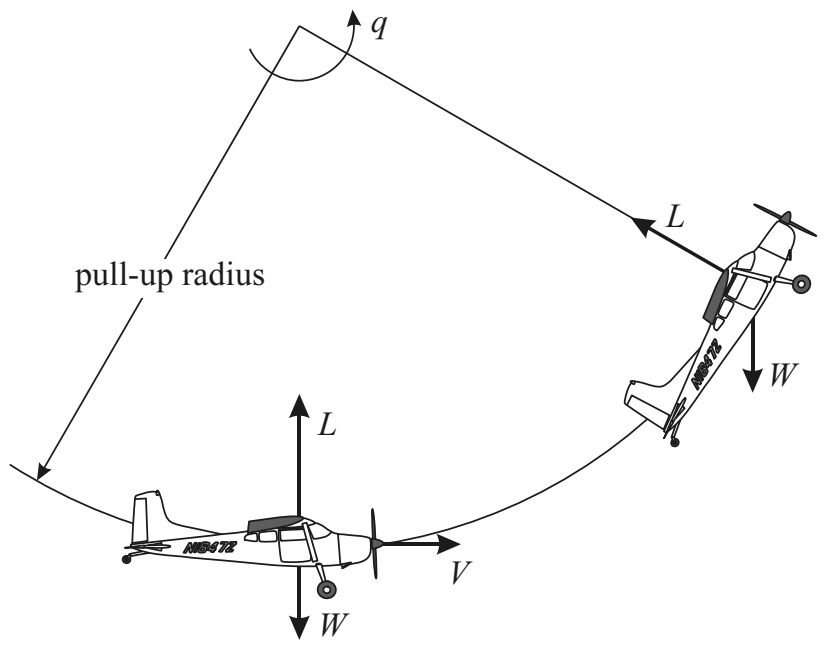

Fig. 1 Quasi-steady pull-up maneuver. 


$$
\left[\begin{array}{ccccc}
\breve{L}_{, \alpha} & 0 & 0 & \breve{L}_{, \delta_{e}} & 0 \\
0 & \breve{Y}_{, \beta} & \breve{Y}_{, \delta_{a}} & 0 & \breve{Y}_{, \delta_{r}} \\
0 & \breve{\ell}, \beta_{, \beta} & \breve{\ell}_{, \delta_{a}} & 0 & \breve{\ell}_{, \delta_{r}} \\
\breve{m}_{, \alpha} & \breve{m}_{, \beta} & 0 & \breve{m}_{, \delta_{e}} & 0 \\
\breve{n}_{, \alpha} & \breve{n}_{, \beta} & \breve{n}_{\delta_{a}} & 0 & \breve{n}_{, \delta_{r}}
\end{array}\right]\left\{\begin{array}{l}
\alpha \\
\beta \\
\delta_{a} \\
\delta_{e} \\
\delta_{r}
\end{array}\right\}=\left\{\begin{array}{c}
1-\breve{L}_{0}+\left(1-\breve{L}_{, \breve{q}}\right)(\breve{L}-1) \\
0 \\
0 \\
-\breve{m}_{0}-\breve{m}_{, \breve{q}}(\breve{L}-1) \\
0
\end{array}\right\}
$$

We see from Eq. (18) that eliminating the bank angle and the rolling and yawing rates from Eq. (15) eliminates the inertial coupling. However, the aerodynamic coupling remains. Hence, both longitudinal and lateral control inputs are required for the quasi-steady pull-up maneuver in airplanes with aerodynamic coupling, such as would be generated by the propeller of a single-engine airplane or other asymmetric aerodynamic loading. In the absence of aerodynamic coupling $\left(\breve{n}_{, \alpha}=\breve{m}_{, \beta}=0\right)$, the second, third, and fifth components of Eq. (18) become trivial and the first and fourth components reduce to

$$
\left[\begin{array}{cc}
\breve{L}_{, \alpha} & \breve{L}_{, \delta_{e}} \\
\breve{m}_{, \alpha} & \breve{m}_{, \delta_{e}}
\end{array}\right]\left\{\begin{array}{c}
\alpha \\
\delta_{e}
\end{array}\right\}=\left\{\begin{array}{c}
1-\breve{L}_{0}+\left(1-\breve{L}_{\breve{q}}\right)(\breve{L}-1) \\
-\breve{m}_{0}-\breve{m}_{, \breve{q}}(\breve{L}-1)
\end{array}\right\}
$$

Equation (19) is readily solved for the angle of attack and elevator deflection required to support a given load factor at $\theta=0$ in the quasi-steady pull-up maneuver with no aerodynamic coupling,

$$
\begin{aligned}
& \alpha=\frac{\left(\breve{L}-\breve{L}_{0}\right) \breve{m}_{, \delta_{e}}+\breve{L}_{, \delta_{e}} \breve{m}_{0}+\left(\breve{L}_{, \delta_{e}} \breve{m}_{, \breve{q}}-\breve{L}_{, \breve{q}} \breve{m}_{, \delta_{e}}\right)(\breve{L}-1)}{\breve{L}_{, \alpha} \breve{m}_{, \delta_{e}}-\breve{L}_{, \delta_{e}} \breve{m}_{, \alpha}} \\
& \delta_{e}=-\frac{\left(\breve{L}-\breve{L}_{0}\right) \breve{m}_{, \alpha}+\breve{L}_{, \alpha} \breve{m}_{0}+\left(\breve{L}_{, \alpha} \breve{m}_{, \breve{q}}-\breve{L}_{, \breve{q}} \breve{m}_{, \alpha}\right)(\breve{L}-1)}{\breve{L}_{, \alpha} \breve{m}_{\delta_{e}}-\breve{L}_{, \delta_{e}} \breve{m}_{, \alpha}}
\end{aligned}
$$

The pitching moment about the CG can be expressed in terms of the pitching moment about the airplane's neutral point $(n p)$, the lift, and the distance that the neutral point lies aft of the $\mathrm{CG}$,

$$
m=m_{n p}-l_{n p} L
$$

In view of Eq. (12), this relation can be nondimensionalized to give

$$
\breve{m} \equiv \frac{m V^{2}}{g^{2} I_{y y}}=\frac{\left(m_{n p}-l_{n p} L\right) V^{2}}{g^{2} I_{y y}}=\breve{m}_{n p}-\frac{W V^{2} l_{n p}}{g^{2} I_{y y}} \breve{L}
$$

Equation (23) suggests the definition for a dynamic length scale ratio

$$
\breve{l}_{n p} \equiv \frac{W V^{2} l_{n p}}{g^{2} I_{y y}}=\frac{V^{2} l_{n p}}{g r_{y y}^{2}}
$$

where $r_{y y}$ is the pitch radius of gyration. By definition, the pitching moment about the neutral point does not vary with small changes in angle of attack. Thus, in the absence of aerodynamic coupling we have

$$
\breve{m}=\breve{m}_{n p}-\breve{l}_{n p} \breve{L}=\breve{m}_{n p 0}+\breve{m}_{n p, \delta_{e}} \delta_{e}+\breve{m}_{n p, \breve{q}} \breve{q}-\breve{l}_{n p}\left(\breve{L}_{0}+\breve{L}_{, \alpha} \alpha+\breve{L}_{, \delta_{e}} \delta_{e}+\breve{L}_{, \breve{q}} \breve{q}\right)
$$

which yields

$$
\breve{m}_{0}=\breve{m}_{n p 0}-\breve{l}_{n p} \breve{L}_{0}, \quad \breve{m}_{, \alpha}=-\breve{l}_{n p} \breve{L}_{, \alpha}, \quad \breve{m}_{, \delta_{e}}=\breve{m}_{n p, \delta_{e}}-\breve{l}_{n p} \breve{L}_{, \delta_{e}}, \quad \breve{m}_{, \breve{q}}=\breve{m}_{n p, \breve{q}}-\breve{l}_{n p} \breve{L}_{, \breve{q}}
$$


Using Eq. (26) in Eqs. (20) and (21) results in

$$
\begin{gathered}
\alpha=\frac{\breve{L}-\breve{L}_{0}-\breve{L}_{, \breve{q}}(\breve{L}-1)}{\breve{L}_{, \alpha}}+\frac{\left[\breve{m}_{n p 0}+\breve{m}_{n p, \breve{q}}(\breve{L}-1)-\breve{l}_{n p} \breve{L}\right] \breve{L}_{, \delta_{e}}}{\breve{L}_{, \alpha} \breve{m}_{n p, \delta_{e}}} \\
\delta_{e}=\frac{\breve{l}_{n p} \breve{L}-\breve{m}_{n p 0}-\breve{m}_{n p, \breve{q}}(\breve{L}-1)}{\breve{m}_{n p, \delta_{e}}}
\end{gathered}
$$

Because steady level flight (trim) can be viewed as a 1-g pull-up maneuver, the elevator angle required for steady level flight can be found as a special case of Eq. (28) with the load factor set to 1.0,

$$
\left(\delta_{e}\right)_{\operatorname{trim}}=\frac{\breve{l}_{n p}-\breve{m}_{n p 0}}{\breve{m}_{n p, \delta_{e}}}=\frac{l_{n p} W-m_{n p 0}}{m_{n p, \delta_{e}}}
$$

Using Eq. (29) in Eq. (28), the elevator angle required to support a quasi-steady pull-up maneuver at $\theta=0$ is

$$
\left(\delta_{e}\right)_{\text {pull-up }}=\left(\delta_{e}\right)_{\text {trim }}+\frac{\breve{l}_{n p}-\breve{m}_{n p, \breve{q}}}{\breve{m}_{n p, \delta_{e}}}(\breve{L}-1)=\left(\delta_{e}\right)_{\text {trim }}+\frac{l_{n p} W-m_{n p, \breve{q}}}{m_{n p, \delta_{e}}}(\breve{L}-1)
$$

The elevator angle per $g$ is found by differentiating Eq. (30) with respect to the load factor, which gives

$$
\left(\frac{\partial \delta_{e}}{\partial \breve{L}}\right)_{\text {pull-up }}=\frac{\breve{l}_{n p}-\breve{m}_{n p, \breve{q}}}{\breve{m}_{n p, \delta_{e}}}=\frac{l_{n p} W-m_{n p, \breve{q}}}{m_{n p, \delta_{e}}}
$$

The stick-fixed maneuver point $(\mathrm{mp})$ for an airplane is defined to be the center of gravity location that would force the elevator angle per $g$ to zero. By definition, $l$ is used here to represent an axial distance measured aft of the CG and $a$ is used to denote an axial distance measured aft of an arbitrary reference point. Thus, we can write $l_{n p} \equiv a_{n p}-\bar{a}$ where an overbar denotes the CG. Equation (31) can then be written as

$$
\left(\frac{\partial \delta_{e}}{\partial \breve{L}}\right)_{\text {pull-up }}=\frac{\left(a_{n p}-\bar{a}\right) W-m_{n p, \breve{q}}}{m_{n p, \delta_{e}}}
$$

With the center of gravity located at the stick-fixed maneuver point and the elevator angle per $g$ set to zero we have $\left(a_{n p}-a_{m p}\right) W-m_{n p, \breve{q}}=0$ or $a_{m p}=a_{n p}-m_{n p, \breve{q}} / W$. Thus, after subtracting $\bar{a}$ from both sides of this latter relation, the distance aft from the actual CG to the stick-fixed maneuver point is determined from

$$
l_{m p}=l_{n p}-\frac{m_{n p, \breve{q}}}{W} \quad \text { or } \quad \breve{l}_{m p}=\breve{l}_{n p}-\breve{m}_{n p, \breve{q}}
$$

where

$$
\breve{l}_{m p} \equiv \frac{W V^{2} l_{m p}}{g^{2} I_{y y}}=\frac{V^{2} l_{m p}}{g r_{y y}^{2}}
$$

The dimensional dynamic derivative $m_{n p, \breve{q}}$ can be expressed in terms of the traditional nondimensional derivative that is commonly determined from wind-tunnel testing. The traditional nondimensional pitch rate is defined as

$$
\bar{q} \equiv q c_{\text {ref }} /(2 V)
$$


Using Eqs. (8) and (34) together with the traditional definition for the moment coefficient yields

$$
m_{n p, \breve{q}} \equiv \frac{1}{2} \rho V^{2} S_{w} c_{\mathrm{ref}} C_{m_{n p}, \breve{q}} \equiv \frac{1}{4} \rho g S_{w} c_{\mathrm{ref}}^{2} C_{m_{n p}, \bar{q}}
$$

Similarly, we have

$$
m_{n p 0} \equiv \frac{1}{2} \rho V^{2} S_{w} c_{\text {ref }} C_{m_{n p} 0}
$$

and

$$
m_{n p, \delta_{e}} \equiv \frac{1}{2} \rho V^{2} S_{w} c_{\mathrm{ref}} C_{m_{n p}, \delta_{e}}
$$

Using Eqs. (36) and (37) in Eq. (29), the elevator angle required for steady level flight can be written as

$$
\left(\delta_{e}\right)_{\text {trim }}=\frac{C_{W}}{c_{\text {ref }} C_{m_{n p}, \delta_{e}}}\left(a_{n p}-\bar{a}\right)-\frac{C_{m_{n p} 0}}{C_{m_{n p}, \delta_{e}}}
$$

If the position of the neutral point and the aerodynamic pitching moment about the neutral point are independent of the position of the CG, Eq. (38) predicts that the elevator deflection required for steady level flight is a linear function of both the weight coefficient and the position of the CG. For a fixed CG located forward of the stickfixed neutral point, the required elevator deflection becomes more negative as the weight coefficient is increased.

Figure 2 shows the elevator deflection required for steady level flight as a function of weight coefficient for a typical single-engine general-aviation airplane with the CG fixed at three different positions. The data used to generate this figure were obtained from Phillips. ${ }^{33}$ The thick red lines show the elevator deflection as predicted from Eq. (38), which neglects the aerodynamic coupling. The thin black lines show the elevator deflection as predicted from Eq. (18) including the aerodynamic coupling. Notice that although some rudder deflection is needed to compensate for the propeller yawing moment, this aerodynamic coupling does not appreciably affect the elevator deflection. The rudder deflection predicted for this airplane from Eq. (18) at a weight coefficient of 1.6 is about -2 degrees. However, the difference between the elevator deflection predicted from Eq. (18) and that predicted from Eq. (38) is less than 0.02 degrees at this weight coefficient. The circular symbols shown in Fig. 2 represent elevator deflections predicted from numerical lifting-line computations. ${ }^{34}$ The deviations between the numerical lifting-line results and the results predicted from Eqs. (18) and (38) are primarily a consequence of the fact that, for deflection angles greater than about 10 degrees, the pitching moment is actually a nonlinear function of elevator deflection. Results similar to those shown in Fig. 2 are presented in Fig. 3 with the elevator angle plotted as a function of the CG location measured aft of the wing quarter chord for three different fixed weight coefficients.

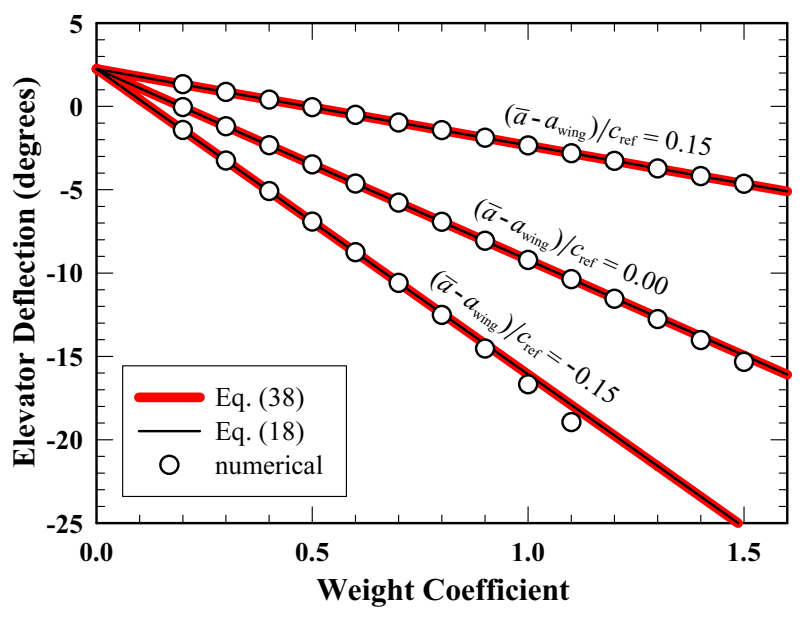

Fig. 2 Elevator deflection for steady level flight as a function of weight coefficient for a typical singleengine general-aviation airplane.

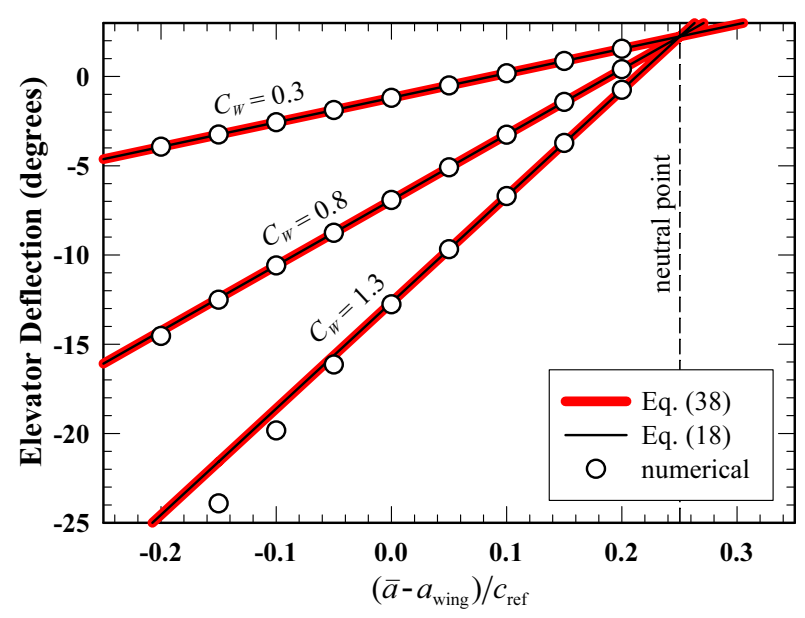

Fig. 3 Elevator deflection for steady level flight as a function of CG location for a typical single-engine general-aviation airplane. 
Note from Eq. (38) and Fig. 3 that the neutral point corresponds to the CG location where the elevator angle required for steady level flight is independent of weight coefficient. From Eq. (38) we see that the change in elevator angle with respect to weight coefficient is a linear function of the CG location

$$
\left(\frac{\partial \delta_{e}}{\partial C_{W}}\right)_{\text {trim }}=\frac{a_{n p}-\bar{a}}{c_{\mathrm{ref}} C_{m_{n p}, \delta_{e}}}
$$

As shown in Fig. 4, the neutral point is the CG location that would force this elevator gradient to zero.

Thus, the axial position of the neutral point can be evaluated by plotting flight-test data in the format of Fig. 2. The slope of the small-angle asymptote for each CG location is evaluated and the results are plotted in the format of Fig. 4. The neutral point is the CG ordinate of the horizontal-axis intercept for the line shown in Fig. 4. The reader should also notice from Eq. (39) that the slope of the line shown in Fig. 4 depends only on the elevator control derivative relative to the neutral point. Differentiating Eq. (39) with respect to $\bar{a}$ and solving for this elevator control derivative yields

$$
C_{m_{n p}, \delta_{e}}=\frac{1}{c_{\mathrm{ref}}} /\left(\frac{\partial^{2} \delta_{e}}{\partial \bar{a} \partial C_{W}}\right)_{\text {trim }}
$$

This relation can be used to evaluate the elevator control derivative relative to the neutral point from flight-test data plotted in the format of Fig. 4. With this elevator control derivative and the location of the neutral point known, the basic moment coefficient about the neutral point, $C_{m_{n p}}$, could be evaluated from the vertical ordinate of the intersection of the lines shown in Fig. 3. From Eq. (38) we obtain

$$
C_{m_{n p} 0}=-C_{m_{n p}, \delta_{e}}\left(\delta_{e}\right)_{\operatorname{trim}}
$$

Thus, we see that the location of the neutral point as well as the elevator control derivative and basic moment coefficient about the neutral point can be determined from flight-test data taken for the elevator angle required to maintain steady level flight.

The flight-test data used to locate the neutral point and evaluate the elevator control derivative relative to the neutral point should be collected for several CG locations within the safe operating range of the airplane. For safety, it is wise to start with the CG located at or near the wing quarter chord and work carefully outward in both directions. For each CG location, the elevator angle should be recorded while the aircraft maintains steady level flight over a range of airspeeds from just above stall to the maximum attainable airspeed. These data are then plotted in the format of Fig. 2, the resulting small-angle slopes are plotted in the format of Fig. 4, and the resulting line is extrapolated to the neutral point.

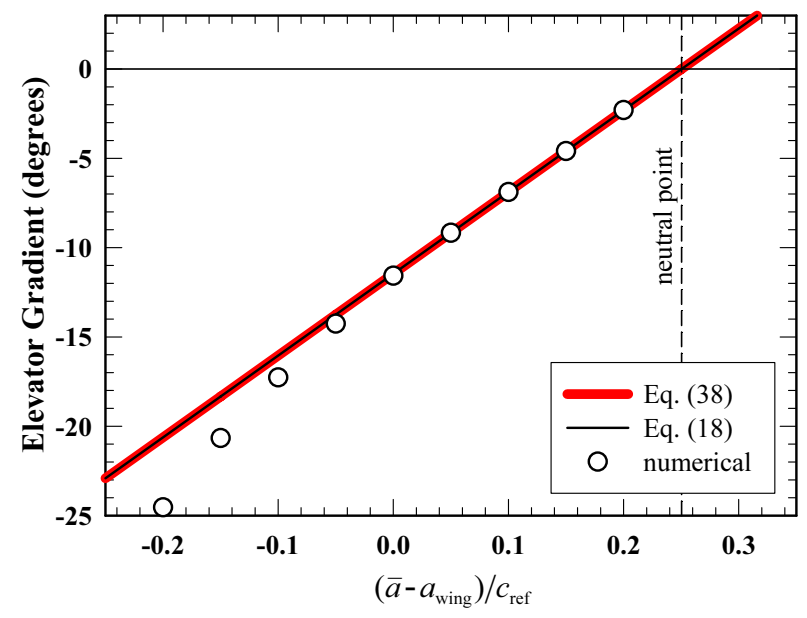

Fig. 4 Elevator gradient with respect to weight coefficient for steady level flight as a function of CG location measured aft of the wing quarter chord for a typical single-engine general-aviation airplane. 
This test procedure is not restricted to UAV applications. It is the established procedure for experimentally determining the neutral point of manned airplanes. ${ }^{35-39}$ However, it should be emphasized that Eq. (38) assumes that the pitching moment is controlled entirely by the elevator and the subscript "trim" denotes steady level flight, not zero control force. Thus, when this procedure is used for a conventional piloted airplane with reversible mechanical controls, all of the associated test data need to be taken at a single trim setting. This is particularly important for airplanes that use a variable stabilizer incidence angle to adjust the control force at trim. Because the pilot needs to supply a continuous control force to maintain steady level flight as the airspeed is changed, a midrange trim setting is most convenient for collecting the complete dataset. This is not a concern for UAV or other fly-by-wire applications, which do not require an aerodynamic trim mechanism for control force adjustment.

Using Eqs. (35)-(38) in Eq. (30), the elevator angle required at $\theta=0$ for the quasi-steady pull-up maneuver can be written as

$$
\left(\delta_{e}\right)_{\text {pull-up }}=\frac{C_{W} \breve{L}\left[\left(a_{n p}-\bar{a}\right) / c_{\text {ref }}\right]-C_{m_{n p} 0}-C_{m_{n p}, \breve{q}}(\breve{L}-1)}{C_{m_{n p}, \delta_{e}}}
$$

Similarly, from Eqs. (31) and (32) we obtain

$$
\begin{gathered}
\left(\frac{\partial \delta_{e}}{\partial \breve{L}}\right)_{\text {pull-up }}=\frac{C_{W}\left[\left(a_{n p}-\bar{a}\right) / c_{\text {ref }}\right]-C_{m_{n p}, \breve{q}}}{C_{m_{n p}, \delta_{e}}} \\
\frac{l_{m p}}{c_{\text {ref }}}=\frac{l_{n p}}{c_{\text {ref }}}-\frac{C_{m_{n p}, \breve{q}}}{C_{W}}
\end{gathered}
$$

All of the aerodynamic coefficients in Eqs. (42)-(44), with the exception of $C_{m_{n p}, \breve{q}}$, can be determined from static measurements combined with flight-test data taken for steady level flight. To evaluate $C_{m_{n p}, \breve{q}}$ experimentally, we must rely on wind-tunnel measurements or flight-test data collected during maneuvering flight.

Equations (42)-(44) may suggest evaluating $C_{m_{n p}, \breve{q}}$ from measurements taken during pull-up maneuvers, as is commonly done with manned airplanes. ${ }^{35-38}$ However, this is not a convenient option for UAVs, because the elevator angle per $g$ is defined for the limiting case of a quasi-steady pull-up maneuver at $\theta=0$, which is not easily replicated with a UAV autopilot. A more practical option for evaluating $C_{m_{n p}, \breve{q}}$ from UAV flight-test data is to use measurements taken during a steady coordinated turn, ${ }^{35-38}$ which is easily maintained with a UAV autopilot.

\section{Steady Coordinated Turn}

The relations presented in Eq. (15) can also be used to evaluate the aerodynamic angles and control surface deflections required to maintain the steady coordinated turn, which is shown in Fig. 5. The angular velocity vector for this steady coordinated turn is constant and parallel to the weight vector. Thus, using the same geometric relations that were used for the weight vector in Eq. (3), the components of the airplane's angular velocity vector in body-fixed coordinates are ${ }^{40}$

$$
\left\{\begin{array}{l}
p \\
q \\
r
\end{array}\right\}=\left\{\begin{array}{c}
-\Omega \sin \theta \\
\Omega \cos \theta \sin \phi \\
\Omega \cos \theta \cos \phi
\end{array}\right\}
$$

where $\Omega$ is the angular velocity magnitude, commonly called the turning rate. For a steady turn, $\Omega, \theta$, and $\phi$ remain constant. However, an additional restriction is needed to account for the fact that the turn is also coordinated.

By definition, a coordinated maneuver is one in which the controls are coordinated so that the vector sum of the airplane's acceleration and the acceleration of gravity produces an apparent body force that falls in the aircraft's plane of symmetry. In other words, the apparent body force has no component in the spanwise direction. From the second component of Eq. (3), this requires

$$
\dot{v}+u r-w p-g \cos \theta \sin \phi=0
$$




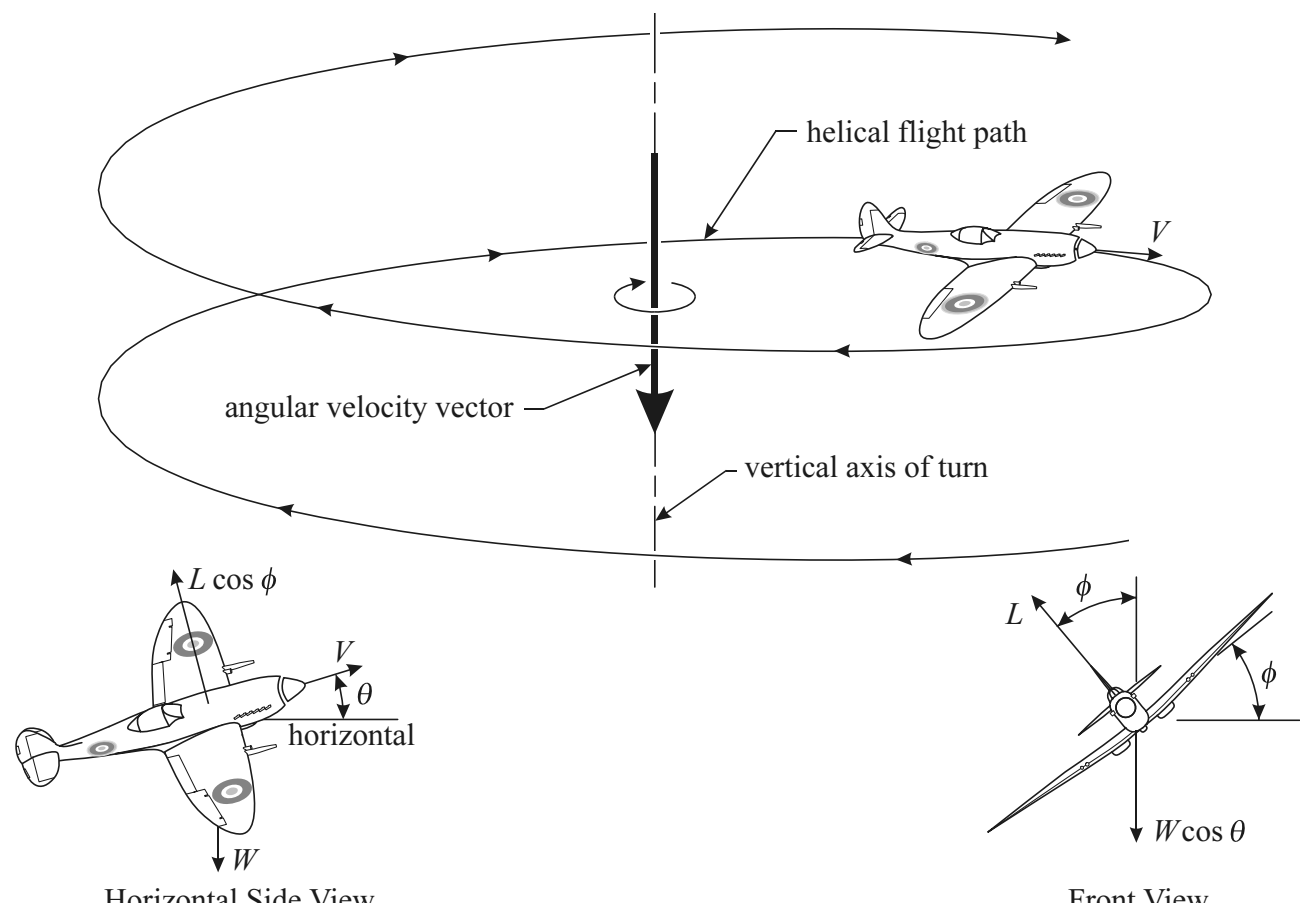

Fig. 5 Steady coordinated turn.

Under the restrictions of steady flight and the small-angle-of-attack approximations ( $\dot{v}=0, u \cong V$, and $w \cong V \alpha$ ), the relations given by Eq. (45) applied to Eq. (46) yield

$$
V \Omega(\cos \theta \cos \phi+\alpha \sin \theta)-g \cos \theta \sin \phi=0
$$

If the rate of climb is small compared with the forward velocity, the second-order term $\alpha \sin \theta$ can be neglected, and after solving for the turning rate, Eq. (47) produces the well-known result

$$
\Omega=(g / V) \tan \phi
$$

Using Eq. (48) in Eq. (45), the body-fixed angular rates for the steady coordinated turn at small climb angles can be written as

$$
\left\{\begin{array}{l}
p \\
q \\
r
\end{array}\right\}=\frac{g}{V}\left\{\begin{array}{c}
-\sin \theta \tan \phi \\
\cos \theta \sin \phi \tan \phi \\
\cos \theta \sin \phi
\end{array}\right\} \quad \text { or } \quad\left\{\begin{array}{l}
\breve{p} \\
\breve{q} \\
\breve{r}
\end{array}\right\}=\left\{\begin{array}{c}
-\sin \theta \tan \phi \\
\cos \theta \sin \phi \tan \phi \\
\cos \theta \sin \phi
\end{array}\right\}
$$

With the application of Eq. (49), the linear system given by Eq. (15) is readily solved for the aerodynamic angles and control surface deflections required to maintain a steady coordinated turn, including the effects of inertial and aerodynamic coupling. However, the angular rates in a coordinated turn are typically small enough so that this longitudinal-lateral coupling has no significant effect on the required elevator deflection. Neglecting all inertial and aerodynamic coupling in Eq. (15) and applying Eq. (49) yields

$$
\left[\begin{array}{ccccc}
\breve{L}_{, \alpha} & 0 & 0 & \breve{L}_{, \delta_{e}} & 0 \\
0 & \breve{Y}_{, \beta} & \breve{Y}_{\delta_{a}} & 0 & \breve{Y}_{, \delta_{r}} \\
0 & \breve{\ell}, \beta_{, \beta} & \ell_{, \delta_{a}} & 0 & \breve{\ell}_{, \delta_{r}} \\
\breve{m}_{, \alpha} & 0 & 0 & \breve{m}_{, \delta_{e}} & 0 \\
0 & \breve{n}_{, \beta} & \breve{n}_{, \delta_{a}} & 0 & \breve{n}_{, \delta_{r}}
\end{array}\right]\left\{\begin{array}{l}
\alpha \\
\beta \\
\delta_{a} \\
\delta_{e} \\
\delta_{r}
\end{array}\right\}=\left\{\begin{array}{c}
{\left[1+\left(1-\breve{L}_{, \breve{q}}\right) \tan ^{2} \phi\right] \cos \theta \cos \phi-\breve{L}_{0}} \\
\breve{Y}_{\breve{p}} \sin \theta \tan \phi-\breve{Y}_{\breve{r}} \cos \theta \sin \phi \\
\breve{\ell}_{, \breve{p}} \sin \theta \tan \phi-\breve{\ell}_{, \breve{r}} \cos \theta \sin \phi \\
-\breve{m}_{, \breve{q}} \cos \theta \sin \phi \tan \phi-\breve{m}_{0} \\
\breve{n}_{, \breve{p}} \sin \theta \tan \phi-\breve{n}_{, \breve{r}} \cos \theta \sin \phi
\end{array}\right\}
$$


Figure 6 shows the control surface deflections required to maintain a steady coordinated turn at constant altitude for the same general-aviation airplane that was used to obtain Figs. 2-4. The thin black lines in Fig. 6 were obtained by using Eq. (49) in Eq. (15), including all inertial and aerodynamic coupling. The thick red lines were obtained from Eq. (50), which neglects all inertial and aerodynamic coupling. Notice that for this airplane, the rudder deflection predicted from Eq. (15) is not symmetric with respect to right and left turns. This is primarily a result of the yawing moment produced by the airplane's propeller. When the airplane is turned either to the right or to the left, additional lift is needed to produce the turning acceleration. As seen in Fig. 6, an increment of up elevator (negative $\delta_{e}$ ) must be applied to increase the angle of attack and generate this added lift. However, this increase in angle of attack also produces an increased propeller yawing moment to the left. This must be countered with an increment of right rudder (negative $\delta_{r}$ ). If the airplane is being turned to the right (positive $\phi$ ), the right rudder needed to compensate for the propeller yawing moment adds to the right rudder needed for the turn. If the airplane is being turned to the left, the right rudder needed to compensate for the propeller yawing moment decreases the left rudder needed for the turn. However, it should be noted that the elevator and aileron deflections are not appreciably affected by this longitudinal-lateral coupling. Thus, for all practical purposes, the elevator deflection required for a coordinated turn can be determined from Eq. (50). Because all longitudinal-lateral coupling was neglected, the first and fourth components of Eq. (50) can be separated from the other components, and for a steady coordinated turn at constant altitude $(\theta=0)$, we obtain

$$
\left[\begin{array}{cc}
\breve{L}_{, \alpha} & \breve{L}_{, \delta_{e}} \\
\breve{m}_{, \alpha} & \breve{m}_{, \delta_{e}}
\end{array}\right]\left\{\begin{array}{c}
\alpha \\
\delta_{e}
\end{array}\right\}=\left\{\begin{array}{c}
\left(1-\breve{L}_{, \breve{q}} \sin ^{2} \phi\right) / \cos \phi-\breve{L}_{0} \\
-\breve{m}_{, \breve{q}} \sin ^{2} \phi / \cos \phi-\breve{m}_{0}
\end{array}\right\}
$$

From the second component of Eq. (13), the load factor for any steady maneuver is

$$
\breve{L}=\cos \theta \cos \phi+\breve{q}-\breve{p} \beta
$$

Using Eq. (49) in Eq. (52) and setting the elevation angle to zero, the load factor for a steady coordinated turn at constant altitude is given by the well-known relation

$$
\breve{L}=\cos \phi+\sin \phi \tan \phi=\left(\cos ^{2} \phi+\sin ^{2} \phi\right) / \cos \phi=1 / \cos \phi
$$

which also yields

$$
\sin ^{2} \phi=\left(\breve{L}^{2}-1\right) / \breve{L}^{2}
$$

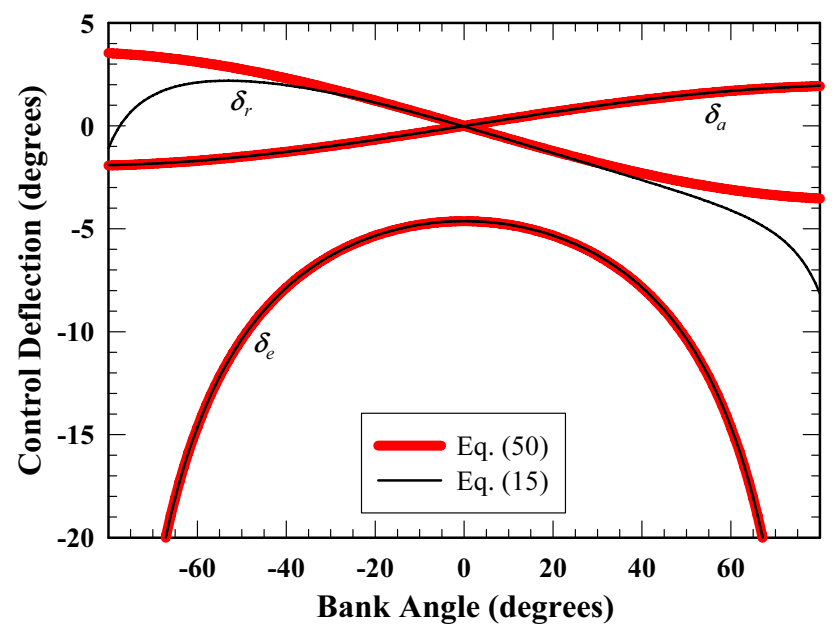

Fig. 6 Control surface deflections for a steady coordinated turn at constant altitude in a typical single-engine general-aviation airplane with a weight coefficient of 0.6 and the $C G$ at the wing quarter chord. 
Using Eqs. (53) and (54) in Eq. (51) results in

$$
\left[\begin{array}{cc}
\breve{L}_{, \alpha} & \breve{L}_{, \delta_{e}} \\
\breve{m}_{, \alpha} & \breve{m}_{, \delta_{e}}
\end{array}\right]\left\{\begin{array}{c}
\alpha \\
\delta_{e}
\end{array}\right\}=\left\{\begin{array}{c}
\breve{L}-\breve{L}_{0}-\breve{L}_{, \breve{q}}(\breve{L}-1 / \breve{L}) \\
-\breve{m}_{0}-\breve{m}_{, \breve{q}}(\breve{L}-1 / \breve{L})
\end{array}\right\}
$$

Following a procedure similar to that used to obtain Eq. (30), the elevator angle required to support a steady coordinated turn at constant altitude can be related to the elevator angle required for steady level flight at the same weight coefficient and the load factor for the coordinated turn. The solution to Eq. (55) is

$$
\begin{gathered}
\alpha=\frac{\left(\breve{L}-\breve{L}_{0}\right) \breve{m}_{, \delta_{e}}+\breve{L}_{, \delta_{e}} \breve{m}_{0}+\left(\breve{L}_{, \delta_{e}} \breve{m}_{, \breve{q}}-\breve{L}_{, \breve{q}} \breve{m}_{, \delta_{e}}\right)(\breve{L}-1 / \breve{L})}{\breve{L}_{, \alpha} \breve{m}_{\delta_{e}}-\breve{L}_{, \delta_{e}} \breve{m}_{, \alpha}} \\
\delta_{e}=-\frac{\left(\breve{L}-\breve{L}_{0}\right) \breve{m}_{, \alpha}+\breve{L}_{, \alpha} \breve{m}_{0}+\left(\breve{L}_{, \alpha} \breve{m}_{, \breve{q}}-\breve{L}_{, \breve{q}} \breve{m}_{, \alpha}\right)(\breve{L}-1 / \breve{L})}{\breve{L}_{, \alpha} \breve{m}_{\delta_{e}}-\breve{L}_{, \delta_{e}} \breve{m}, \alpha_{, \alpha}}
\end{gathered}
$$

After applying Eq. (26) to express the pitching moment about the CG in terms of the pitching moment about the neutral point, we have

$$
\begin{gathered}
\alpha=\frac{\breve{L}-\breve{L}_{0}-\breve{L}_{\breve{q}}(\breve{L}-1 / \breve{L})}{\breve{L}_{, \alpha}}+\frac{\left[\breve{m}_{n p 0}+\breve{m}_{n p, \breve{q}}(\breve{L}-1 / \breve{L})-\breve{l}_{n p} \breve{L}\right] \breve{L}_{, \delta_{e}}}{\breve{L}_{, \alpha} \breve{m}_{n p, \delta_{e}}} \\
\delta_{e}=\frac{\breve{l}_{n p} \breve{L}-\breve{m}_{n p 0}-\breve{m}_{n p, \breve{q}}(\breve{L}-1 / \breve{L})}{\breve{m}_{n p, \delta_{e}}}
\end{gathered}
$$

Using Eq. (29) in Eq. (59) and then applying Eqs. (23) and (24), the elevator angle required to support a given load factor in a steady coordinated turn at constant altitude can be written as

$$
\left(\delta_{e}\right)_{\mathrm{turn}}=\left(\delta_{e}\right)_{\mathrm{trim}}+\frac{\breve{l}_{n p}(\breve{L}-1)-\breve{m}_{n p, \breve{q}}(\breve{L}-1 / \breve{L})}{\breve{m}_{n p, \delta_{e}}}=\left(\delta_{e}\right)_{\text {trim }}+\frac{l_{n p} W(\breve{L}-1)-m_{n p, \breve{q}}(\breve{L}-1 / \breve{L})}{m_{n p, \delta_{e}}}
$$

From a comparison of Eqs. (30) and (60) we see that these relations are similar but not identical. The elevator angle required to support a given load factor in a steady coordinated turn at constant altitude is not the same as that required to support the same load factor in a quasi-steady pull-up maneuver. Figure 7 shows the elevator angle increment relative to steady level flight, which is required to support a given load factor in a steady coordinated turn at constant altitude. The results shown in Fig. 7 are for the same airplane that was used to obtain the results plotted in Figs. 2-4 and 6. The thick red lines were obtained from Eq. (60), which neglects the effects of longitudinallateral coupling. The thin black lines include the effects of longitudinal-lateral coupling as predicted by using Eq. (49) in Eq. (15). For comparison, the dashed lines in Fig. 7 show similar results for the quasi-steady pull-up maneuver as predicted from Eq. (30).

The larger elevator deflection for the coordinated turn is required to support a larger pitch rate. From Eq. (17), the dynamic pitch rate for the quasi-steady pull-up maneuver at $\theta=0$ is

$$
\breve{q}=\breve{L}-1
$$

Using Eqs. (53) and (54) in Eq. (49) gives the dynamic pitch rate for the steady coordinated turn at $\theta=0$ as

$$
\breve{q}=\sin ^{2} \phi / \cos \phi=\breve{L}-1 / \breve{L}
$$

This translates to a $50 \%$ increase in pitch rate for a $2-g$ coordinated turn, relative to that for a $2-g$ pull-up maneuver. 


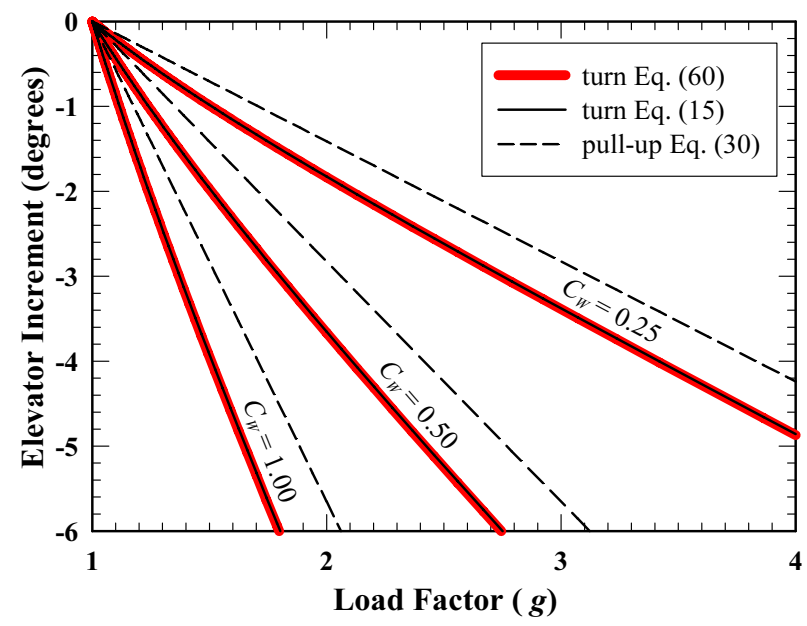

Fig. 7 Elevator increment from steady level flight as a function of load factor for a typical single-engine general-aviation airplane with the CG located to give a static margin of $5 \%$.

From Eq. (60), we see that the elevator deflection required to support a steady coordinated turn at constant altitude can be conveniently divided into three components,

$$
\left(\delta_{e}\right)_{\mathrm{turn}}=\left(\delta_{e}\right)_{\mathrm{trim}}+\left(\Delta \delta_{e}\right)_{\breve{L}}+\left(\Delta \delta_{e}\right)_{\breve{q}}
$$

The first component on the right-hand side of Eq. (63) is the elevator deflection required to support the airplane's weight in steady level flight, which from Eqs. (29) and (38) is

$$
\left(\delta_{e}\right)_{\text {trim }}=\frac{\breve{l}_{n p}-\breve{m}_{n p 0}}{\breve{m}_{n p, \delta_{e}}}=\frac{l_{n p} W-m_{n p 0}}{m_{n p, \delta_{e}}}=\frac{\left(l_{n p} / c_{\mathrm{ref}}\right) C_{W}-C_{m_{n p} 0}}{C_{m_{n p}, \delta_{e}}}
$$

The second component on the right-hand side of Eq. (63) is defined to be the elevator increment required to support the airplane's normal acceleration,

$$
\left(\Delta \delta_{e}\right)_{\breve{L}} \equiv \frac{\breve{l}_{n p}(\breve{L}-1)}{\breve{m}_{n p, \delta_{e}}} \equiv \frac{l_{n p} W(\breve{L}-1)}{m_{n p, \delta_{e}}} \equiv \frac{l_{n p} C_{W}(\breve{L}-1)}{c_{\mathrm{ref}} C_{m_{n p}, \delta_{e}}}
$$

The third component on the right-hand side of Eq. (63) is defined to be the elevator increment required to support the airplane's pitch rate,

$$
\left(\Delta \delta_{e}\right)_{\breve{q}} \equiv-\frac{\breve{m}_{n p, \breve{q}}(\breve{L}-1 / \breve{L})}{\breve{m}_{n p, \delta_{e}}} \equiv-\frac{m_{n p, \breve{q}}(\breve{L}-1 / \breve{L})}{m_{n p, \delta_{e}}} \equiv-\frac{C_{m_{n p}, \breve{q}}(\breve{L}-1 / \breve{L})}{C_{m_{n p}, \delta_{e}}}
$$

As previously discussed, the location of the neutral point as well as the elevator control derivative and basic moment coefficient relative to the neutral point can be determined from flight-test data collected during steady level flight. Thus, the elevator increment defined by Eq. (66) could be determined from measurements of the elevator deflection and load factor taken in a steady coordinated turn at constant altitude combined with knowledge of other parameters, which can be determined from static measurements and flight-test data collected in steady level flight. Thus, an alternate and more useful definition for the elevator increment defined by Eq. (66) is

$$
\left(\Delta \delta_{e}\right)_{\breve{q}} \equiv\left(\delta_{e}\right)_{\text {turn }}-\left(\delta_{e}\right)_{\text {trim }}-\frac{l_{n p} C_{W}(\breve{L}-1)}{c_{\text {ref }} C_{m_{n p}, \delta_{e}}}
$$

where all elevator deflections in Eq. (67) are for the same weight coefficient and CG location. 
For convenience, we will now use Eq. (67) to define what we shall refer to as the turn damping ratio,

$$
R_{\breve{q}} \equiv \frac{C_{m_{n p}, \delta_{e}}\left(\Delta \delta_{e}\right)_{\breve{q}}}{C_{W}} \equiv \frac{C_{m_{n p}, \delta_{e}}\left[\left(\delta_{e}\right)_{\mathrm{turn}}-\left(\delta_{e}\right)_{\mathrm{trim}}\right]}{C_{W}}-\frac{l_{n p}}{C_{\mathrm{ref}}}(\breve{L}-1)
$$

By definition, the turn damping ratio is the pitching-moment-coefficient increment relative to the neutral point, per unit weight coefficient, which is required to support the dynamic pitch rate in a steady coordinated turn at constant altitude. Using Eq. (66) in Eq. (68) and applying Eq. (62) yields the alternate definitions

$$
R_{\breve{q}} \equiv-\frac{C_{m_{n p}, \breve{q}}}{C_{W}}(\breve{L}-1 / \breve{L}) \equiv-\frac{C_{m_{n p}, \breve{q}}}{C_{W}} \breve{q}
$$

Comparing Eqs. (44) and (69), we see that the location of the stick-fixed maneuver point can be expressed in terms of the location of the stick-fixed neutral point and the change in turn damping ratio with respect to dynamic pitch rate,

$$
\frac{l_{m p}}{c_{\text {ref }}}=\frac{l_{n p}}{c_{\text {ref }}}-\frac{C_{m_{n p}, \breve{q}}}{C_{W}}=\frac{l_{n p}}{c_{\text {ref }}}+\frac{\partial R_{\breve{q}}}{\partial \breve{q}}
$$

Figure 8 shows the turn damping ratio plotted as a function of the dynamic pitch rate for the same airplane that was used to obtain Figs. 2-4 and 6-7. The solid line in Fig. 8 was obtained from Eq. (69). All of the symbols shown in Fig. 8 represent results obtained from numerical lifting-line computations. ${ }^{34}$ The circular, square, and diamond-shaped symbols represent results obtained at weight coefficients of $0.25,0.50$, and 1.00 , respectively. The filled symbols are for results obtained with the CG located at the wing quarter chord and the open symbols represent results obtained with the CG located at the $35 \%$ chord. These CG locations correspond to static margins of about 25 and 15 percent, respectively. The deviations between some of the numerical lifting-line predictions and Eq. (69) are primarily a result of the reduction in elevator effectiveness, which occurs at deflection angles greater than about 10 degrees. Because the elevator deflection magnitude increases as the weight coefficient is increased and as the CG is moved forward, this deviation is greatest for the highest weight coefficients at the most forward CG locations.

Once the location of the neutral point and the elevator control derivative relative to the neutral point have been determined from flight-test data collected during steady level flight, Eqs. (62) and (68) can be used to determine the dynamic pitch rate and turn damping ratio from measurements of the load factor and elevator deflection taken during a steady coordinated turn at constant altitude. Such data should be collected over a range of airspeeds and bank angles for several different CG locations within the safe operating range of the airplane. These data should be

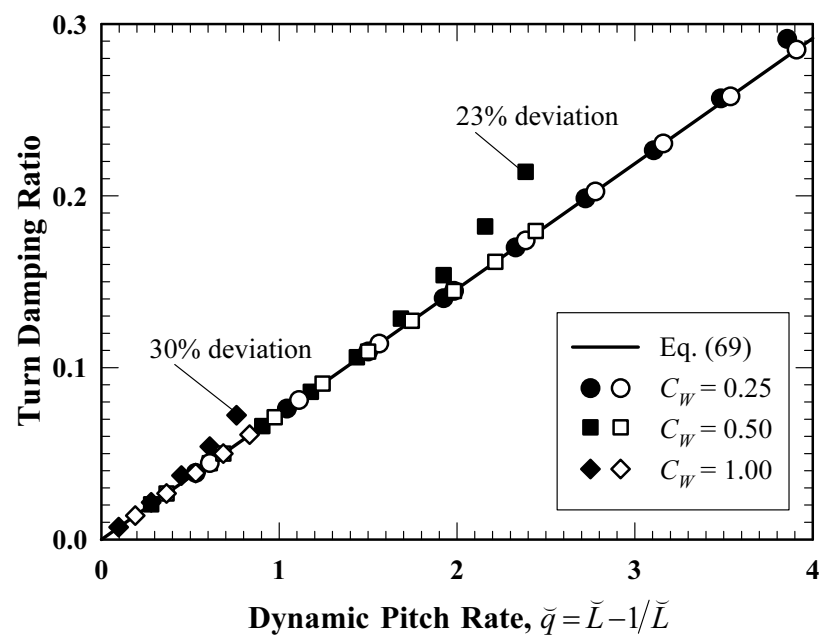

Fig. 8 Turn damping ratio defined by Eq. (68) as a function of dynamic pitch rate for a typical single-engine general-aviation airplane with the CG located at $25 \%$ and $35 \%$ of the wing chord. 
collected while the UAV autopilot is maintaining constant airspeed, altitude, and load factor, as well as a zero spanwise acceleration component. The resulting data are plotted in the format of Fig. 8 and the change in the turn damping ratio with respect to dynamic pitch rate is determined from the slope of the small-angle asymptote. The stick-fixed maneuver point can then be located from Eq. (70).

\section{Mass Property Relations}

As seen from Eq. (2), the control anticipation parameter varies with CG location through its dependence on both the maneuver margin and the pitch radius of gyration. For the purpose of flight testing, the axial position of the CG is commonly varied by carrying some type of ballast, which can be shifted forward or aft to move the CG. This redistribution of mass changes both the maneuver margin and the pitch radius of gyration.

If we let $W_{e}$ and $W_{b}$ denote the empty weight of the airplane without the ballast and the weight of the ballast, respectively, then the axial and normal coordinates of the centers of gravity for the empty airplane and the ballast are defined by the integrals

$$
\bar{x}_{e} \equiv \frac{1}{W_{e}} \iiint_{W_{e}} x d W_{e}, \quad \bar{z}_{e} \equiv \frac{1}{W_{e}} \iiint_{W_{e}} z d W_{e}, \quad \bar{x}_{b} \equiv \frac{1}{W_{b}} \iiint_{W_{b}} x d W_{b}, \quad \bar{z}_{b} \equiv \frac{1}{W_{b}} \iiint_{W_{b}} z d W_{b}
$$

The pitching moments of inertia for the empty airplane and the ballast about their individual centers of gravity are

$$
\left(I_{y y}\right)_{e} \equiv \frac{1}{g} \iint_{W_{e}}\left[\left(x-\bar{x}_{e}\right)^{2}+\left(z-\bar{z}_{e}\right)^{2}\right] d W_{e}, \quad\left(I_{y y}\right)_{b} \equiv \frac{1}{g} \iiint_{W_{b}}\left[\left(x-\bar{x}_{b}\right)^{2}+\left(z-\bar{z}_{b}\right)^{2}\right] d W_{b}
$$

or after expanding the quadratics and applying Eq. (71)

$$
\begin{aligned}
\left(I_{y y}\right)_{e} & =\frac{1}{g} \iiint_{W_{e}}\left(x^{2}-2 x \bar{x}_{e}+\bar{x}_{e}^{2}+z^{2}-2 z \bar{z}_{e}+\bar{z}_{e}^{2}\right) d W_{e} & \left(I_{y y}\right)_{b} & =\frac{1}{g} \iiint_{W_{b}}\left(x^{2}-2 x \bar{x}_{b}+\bar{x}_{b}^{2}+z^{2}-2 z \bar{z}_{b}+\bar{z}_{b}^{2}\right) d W_{b} \\
& =\frac{1}{g} \iiint_{W_{e}}\left(x^{2}+z^{2}\right) d W_{e}-\left(\bar{x}_{e}^{2}+\bar{z}_{e}^{2}\right) W_{e} / g, & & =\frac{1}{g} \iiint_{W_{b}}\left(x^{2}+z^{2}\right) d W_{b}-\left(\bar{x}_{b}^{2}+\bar{z}_{b}^{2}\right) W_{b} / g
\end{aligned}
$$

The axial and normal coordinates of the combined CG for the airplane with ballast are located from

$$
\bar{x}=\frac{\bar{x}_{e} W_{e}+\bar{x}_{b} W_{b}}{W_{e}+W_{b}}, \quad \bar{z}=\frac{\bar{z}_{e} W_{e}+\bar{z}_{b} W_{b}}{W_{e}+W_{b}}
$$

and the pitching moment of inertia for the airplane with ballast about the combined CG is

$$
\begin{aligned}
I_{y y} & \equiv \frac{1}{g} \iiint_{W_{e}}\left[(x-\bar{x})^{2}+(z-\bar{z})^{2}\right] d W_{e}+\frac{1}{g} \iiint_{W_{b}}\left[(x-\bar{x})^{2}+(z-\bar{z})^{2}\right] d W_{b} \\
& =\frac{1}{g} \iiint_{W_{e}}\left(x^{2}-2 x \bar{x}+\bar{x}^{2}+z^{2}-2 z \bar{z}+\bar{z}^{2}\right) d W_{e}+\frac{1}{g} \iiint_{W_{b}}\left(x^{2}-2 x \bar{x}+\bar{x}^{2}+z^{2}-2 z \bar{z}+\bar{z}^{2}\right) d W_{b} \\
& =\frac{1}{g} \iiint_{W_{e}}\left(x^{2}+z^{2}\right) d W_{e}-\left(\bar{x}^{2}+\bar{z}^{2}\right) W_{e} / g+\frac{1}{g} \iiint_{W_{b}}\left(x^{2}+z^{2}\right) d W_{b}-\left(\bar{x}^{2}+\bar{z}^{2}\right) W_{b} / g
\end{aligned}
$$

After using Eq. (73) to eliminate the integrals from Eq. (75) and rearranging, we have

$$
\begin{array}{r}
I_{y y}=\left(I_{y y}\right)_{e}+\left(I_{y y}\right)_{b}+\left(\bar{x}_{e}^{2}-\bar{x}^{2}+\bar{z}_{e}^{2}-\bar{z}^{2}\right) W_{e} / g+\left(\bar{x}_{b}^{2}-\bar{x}^{2}+\bar{z}_{b}^{2}-\bar{z}^{2}\right) W_{b} / g \\
=\left(I_{y y}\right)_{e}+\left(I_{y y}\right)_{b}+\left[\left(\bar{x}_{e}+\bar{x}\right)\left(\bar{x}_{e}-\bar{x}\right)+\left(\bar{z}_{e}+\bar{z}\right)\left(\bar{z}_{e}-\bar{z}\right)\right] W_{e} / g \\
+\left[\left(\bar{x}_{b}+\bar{x}\right)\left(\bar{x}_{b}-\bar{x}\right)+\left(\bar{z}_{b}+\bar{z}\right)\left(\bar{z}_{b}-\bar{z}\right)\right] W_{b} / g
\end{array}
$$


Equation (74) is easily rearranged to give

$$
\left(\bar{x}_{b}-\bar{x}\right) W_{b}=-\left(\bar{x}_{e}-\bar{x}\right) W_{e}, \quad\left(\bar{z}_{b}-\bar{z}\right) W_{b}=-\left(\bar{z}_{e}-\bar{z}\right) W_{e}
$$

Using Eq. (77) in Eq. (76) and rearranging yields

$$
I_{y y}=\left(I_{y y}\right)_{e}+\left(I_{y y}\right)_{b}+\left[\left(\bar{x}_{b}-\bar{x}_{e}\right)\left(\bar{x}-\bar{x}_{e}\right)+\left(\bar{z}_{b}-\bar{z}_{e}\right)\left(\bar{z}-\bar{z}_{e}\right)\right] W_{e} / g
$$

By rearranging Eq. (74) in a different manner we can also obtain

$$
\bar{x}_{b}-\bar{x}_{e}=\frac{\left(\bar{x}-\bar{x}_{e}\right)\left(W_{e}+W_{b}\right)}{W_{b}}, \quad \bar{z}_{b}-\bar{z}_{e}=\frac{\left(\bar{z}-\bar{z}_{e}\right)\left(W_{e}+W_{b}\right)}{W_{b}}
$$

Recognizing that the total gross weight of the airplane is simply the sum of the empty weight and the weight of the ballast, $W=W_{e}+W_{b}$, and using Eq. (79) in Eq. (78) yields

$$
I_{y y}=\left(I_{y y}\right)_{e}+\left(I_{y y}\right)_{b}+\left[\left(\bar{x}-\bar{x}_{e}\right)^{2}+\left(\bar{z}-\bar{z}_{e}\right)^{2}\right](W / g)\left(W_{e} / W_{b}\right)
$$

If the loaded moment of inertia is determined for one particular CG location, the sum of the first two terms on the right-hand side of Eq. (80) can be related to this known moment of inertia and CG location. Rearranging Eq. (80) and evaluating the result at CG-location 1 , we obtain

$$
\left(I_{y y}\right)_{e}+\left(I_{y y}\right)_{b}=\left(I_{y y}\right)_{1}-\left[\left(\bar{x}_{1}-\bar{x}_{e}\right)^{2}+\left(\bar{z}_{1}-\bar{z}_{e}\right)^{2}\right](W / g)\left(W_{e} / W_{b}\right)
$$

Using Eq. (81) in Eq. (80), the loaded moment of inertia at any other CG location can be determined from

$$
I_{y y}=\left(I_{y y}\right)_{1}+\left[\left(\bar{x}-\bar{x}_{e}\right)^{2}-\left(\bar{x}_{1}-\bar{x}_{e}\right)^{2}+\left(\bar{z}-\bar{z}_{e}\right)^{2}-\left(\bar{z}_{1}-\bar{z}_{e}\right)^{2}\right](W / g)\left(W_{e} / W_{b}\right)
$$

The relation given by Eq. (82) is based on a reference loaded moment of inertia evaluated at an arbitrary CG location and it accounts for an arbitrary shift in the position of the ballast. This result is simplified if the reference CG location for the loaded airplane is chosen to have the same axial position as that for the empty airplane. If we also restrict the flight testing to include only axial shifts in the position of the ballast, then Eq. (82) becomes

$$
I_{y y}=\left(I_{y y}\right)_{0}+\left(\bar{x}-\bar{x}_{e}\right)^{2}(W / g)\left(W_{e} / W_{b}\right)
$$

where $\left(I_{y y}\right)_{0}$ is the loaded moment of inertia with the CG located at the same axial position as that of the empty airplane.

\section{Experimental Determination of the Radius of Gyration}

Filar pendulums have been used for the measurement of aircraft mass moments of inertia since the early days of aviation. ${ }^{41-47}$ The simplest and most commonly used by the aircraft community is the bifilar pendulum, ${ }^{48}$ which uses only two supporting cables or strings. Although the usual analysis of the bifilar pendulum is comparatively simple, obtaining accurate results from this simplified analysis requires locating the CG midway between supporting cables or strings. An off-center CG results in precessional motion in addition to the fundamental rotational motion about the vertical axis passing through the CG. Such precessional motion is not induced by an off-center CG when the trifilar pendulum ${ }^{49}$ is used.

Components of the mass moment of inertia tensor for any object can be determined by hanging the object to create a trifilar torsional pendulum as shown in Fig. 9. The object is suspended from three long parallel cables or strings of lengths $s_{1}, s_{2}$, and $s_{3}$. One end of each string is attached to the object and the other end is attached to a rigid supporting member. It is critical to locate the attachments points so that the strings are parallel. 


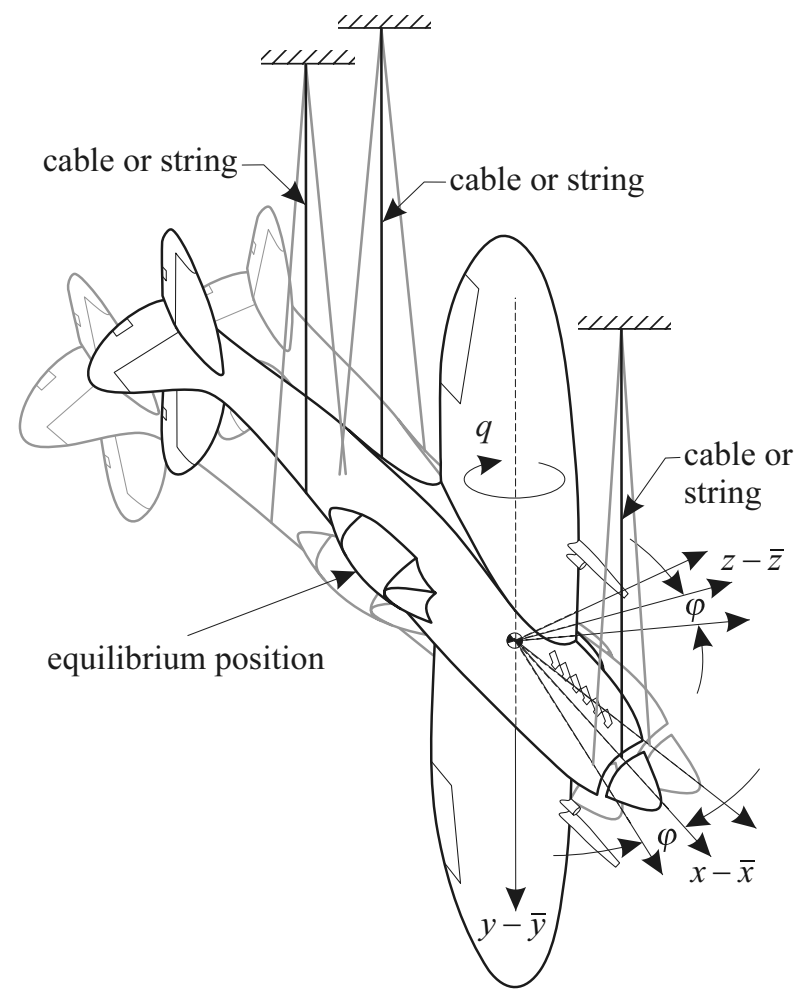

Fig. 9 Aircraft suspended as a trifilar pendulum.

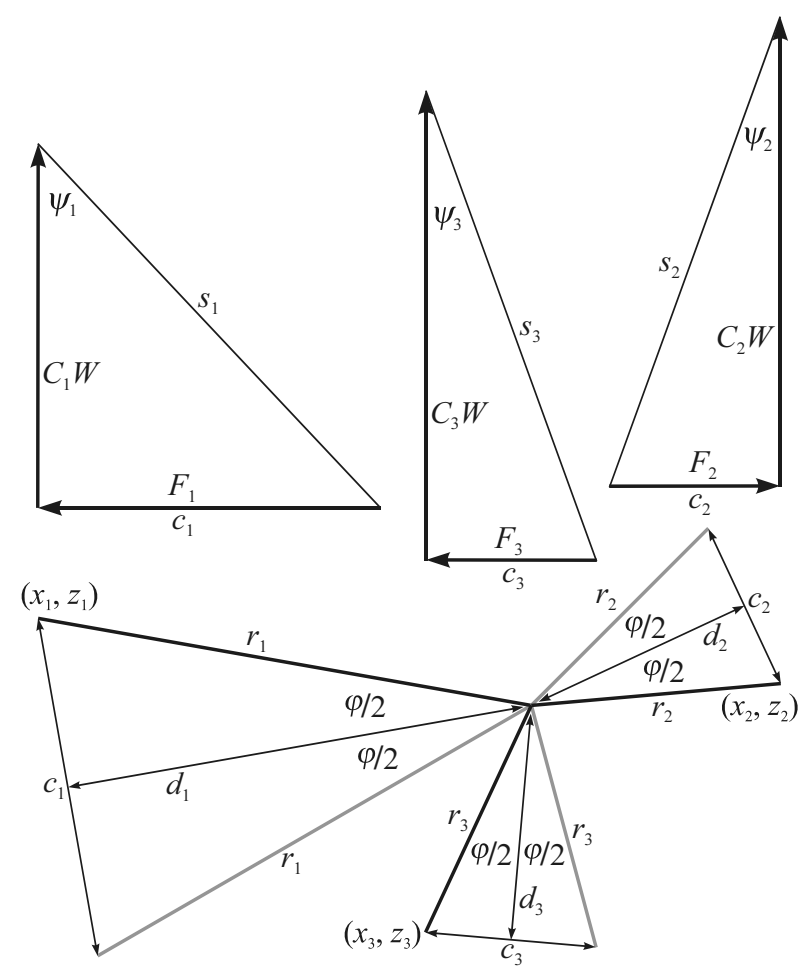

Fig. 10 Trifilar pendulum geometry.

The vertical axis passing through the CG is located between the strings a distance $r_{1}$ from string 1 , a distance $r_{2}$ from string 2, and a distance $r_{3}$ from string 3 . As the object rotates through a small angle $\varphi$ about the vertical axis passing through the CG, the lower attachment points for strings 1, 2, and 3 move through small arcs with horizontal chordlength projections of $c_{1}, c_{2}$, and $c_{3}$, respectively. At the beginning of the arcs, all three strings are vertical. At the end of the arcs, the strings make angles $\psi_{1}, \psi_{2}$, and $\psi_{3}$ with the vertical.

From the geometry shown schematically in Fig. 10, we can write the horizontal chord-length projections in terms of the angles that the strings make with the vertical, $\psi_{1}, \psi_{2}$, and $\psi_{3}$, and in terms of the pendulum rotation angle, $\varphi$,

$$
c_{1}=s_{1} \sin \psi_{1}=2 r_{1} \sin (\varphi / 2), \quad c_{2}=s_{2} \sin \psi_{2}=2 r_{2} \sin (\varphi / 2), \quad c_{3}=s_{3} \sin \psi_{3}=2 r_{3} \sin (\varphi / 2)
$$

Thus, the angles that the strings make with the vertical, $\psi_{1}, \psi_{2}$, and $\psi_{3}$, can be related to the rotation angle, $\varphi$,

$$
\sin \psi_{1}=\frac{2 r_{1}}{s_{1}} \sin (\varphi / 2), \quad \sin \psi_{2}=\frac{2 r_{2}}{s_{2}} \sin (\varphi / 2), \quad \sin \psi_{3}=\frac{2 r_{3}}{s_{3}} \sin (\varphi / 2)
$$

Denoting the vertical components of string tension divided by the weight as $C_{1}, C_{2}$, and $C_{3}$, the restoring forces at the lower string attachment points divided by the weight are

$$
\frac{F_{1}}{W}=C_{1} \tan \psi_{1}=C_{1} \frac{\sin \psi_{1}}{\cos \psi_{1}}, \quad \frac{F_{2}}{W}=C_{2} \tan \psi_{2}=C_{2} \frac{\sin \psi_{2}}{\cos \psi_{2}}, \quad \frac{F_{3}}{W}=C_{3} \tan \psi_{3}=C_{3} \frac{\sin \psi_{3}}{\cos \psi_{3}}
$$

The total restoring moment is the sum of the restoring forces each multiplied by the associated perpendicular moment arm,

$$
M=d_{1} F_{1}+d_{2} F_{2}+d_{3} F_{3}
$$


where from the geometry shown in Fig. 10,

$$
d_{1}=r_{1} \cos (\varphi / 2), \quad d_{2}=r_{2} \cos (\varphi / 2), \quad d_{3}=r_{3} \cos (\varphi / 2)
$$

Using Eqs. (85), (86) and (88) in Eq. (87) yields

$$
M=2\left(\frac{r_{1}^{2} C_{1}}{s_{1} \cos \psi_{1}}+\frac{r_{2}^{2} C_{2}}{s_{2} \cos \psi_{2}}+\frac{r_{3}^{2} C_{3}}{s_{3} \cos \psi_{3}}\right) W \cos (\varphi / 2) \sin (\varphi / 2)
$$

or after applying the half-angle identity, $\sin \varphi=2 \cos (\varphi / 2) \sin (\varphi / 2)$,

$$
M=\left(\frac{r_{1}^{2} C_{1}}{s_{1} \cos \psi_{1}}+\frac{r_{2}^{2} C_{2}}{s_{2} \cos \psi_{2}}+\frac{r_{3}^{2} C_{3}}{s_{3} \cos \psi_{3}}\right) W \sin \varphi
$$

If the strings are long and the rotation angle is small, the vertical components of acceleration can be neglected and the vertical components of string tension support only the weight. If the position of the CG is known, the weight supported by each string can be related to the total weight, $W$, and the coordinates of the string attachment points, $\left(x_{1}, z_{1}\right),\left(x_{2}, z_{2}\right)$, and $\left(x_{3}, z_{3}\right)$. Summing the $y$-force components and the $x$ - and $z$-moment components yields

$$
\left[\begin{array}{ccc}
1 & 1 & 1 \\
\left(x_{1}-\bar{x}\right) & \left(x_{2}-\bar{x}\right) & \left(x_{3}-\bar{x}\right) \\
\left(z_{1}-\bar{z}\right) & \left(z_{2}-\bar{z}\right) & \left(z_{3}-\bar{z}\right)
\end{array}\right]\left\{\begin{array}{l}
C_{1} W \\
C_{2} W \\
C_{3} W
\end{array}\right\}=\left\{\begin{array}{l}
W \\
0 \\
0
\end{array}\right\}
$$

or

$$
\left[\begin{array}{ccc}
1 & 1 & 1 \\
\left(x_{1}-\bar{x}\right) & \left(x_{2}-\bar{x}\right) & \left(x_{3}-\bar{x}\right) \\
\left(z_{1}-\bar{z}\right) & \left(z_{2}-\bar{z}\right) & \left(z_{3}-\bar{z}\right)
\end{array}\right]\left\{\begin{array}{l}
C_{1} \\
C_{2} \\
C_{3}
\end{array}\right\}=\left\{\begin{array}{l}
1 \\
0 \\
0
\end{array}\right\}
$$

If the weight supported by each string is experimentally determined, the position of the CG can be related to the positions of the strings. By rearranging the last two components of Eq. (92) and applying the relation specified by the first component of Eq. (92), we obtain

$$
\left\{\begin{array}{l}
\bar{x} \\
\bar{z}
\end{array}\right\}=\frac{1}{C_{1}+C_{2}+C_{3}}\left\{\begin{array}{l}
x_{1} C_{1}+x_{2} C_{2}+x_{3} C_{3} \\
z_{1} C_{1}+z_{2} C_{2}+z_{3} C_{3}
\end{array}\right\}=\left\{\begin{array}{l}
x_{1} C_{1}+x_{2} C_{2}+x_{3} C_{3} \\
z_{1} C_{1}+z_{2} C_{2}+z_{3} C_{3}
\end{array}\right\}
$$

With the weight supported by each string and the position of the CG known, the distance from each string to the CG is easily determined from

$$
\left\{\begin{array}{l}
r_{1}^{2} \\
r_{2}^{2} \\
r_{3}^{2}
\end{array}\right\}=\left\{\begin{array}{l}
\left(x_{1}-\bar{x}\right)^{2}+\left(z_{1}-\bar{z}\right)^{2} \\
\left(x_{2}-\bar{x}\right)^{2}+\left(z_{2}-\bar{z}\right)^{2} \\
\left(x_{3}-\bar{x}\right)^{2}+\left(z_{3}-\bar{z}\right)^{2}
\end{array}\right\}
$$

If the strings are long and the rotation angle is small we can use the small angle approximations, $\sin \varphi \cong \varphi$ and $\cos \psi_{1} \cong \cos \psi_{2} \cong \cos \psi_{3} \cong 1$. From Eq. (90), this yields the linear restoring moment

$$
M=\left(\frac{r_{1}^{2} C_{1}}{s_{1}}+\frac{r_{2}^{2} C_{2}}{s_{2}}+\frac{r_{3}^{2} C_{3}}{s_{3}}\right) W \varphi
$$


When this trifilar pendulum is given a small angular displacement and then released, it will oscillate in rotation about the vertical axis passing through the CG. Neglecting all aerodynamic forces, Newton's second law for this trifilar pendulum requires

$$
I_{y y} \frac{d^{2} \varphi}{d t^{2}}+\left(\frac{r_{1}^{2} C_{1}}{s_{1}}+\frac{r_{2}^{2} C_{2}}{s_{2}}+\frac{r_{3}^{2} C_{3}}{s_{3}}\right) W \varphi=0
$$

The solution to this second-order differential equation predicts undamped oscillations with a natural frequency of

$$
\omega_{n}=\sqrt{\frac{W}{I_{y y}}\left(\frac{r_{1}^{2} C_{1}}{s_{1}}+\frac{r_{2}^{2} C_{2}}{s_{2}}+\frac{r_{3}^{2} C_{3}}{s_{3}}\right)}
$$

Of course, in any real experiment there will be damping. From Eq. (97) we see that long strings will produce low-frequency oscillations, which result in light damping. By recording the angular position, velocity, or acceleration as a function of time, we can determine both the damped frequency, $\omega_{d}$, and damping rate, $\sigma$. The undamped natural frequency and damping ratio can then be determined from

$$
\omega_{n}=\sqrt{\omega_{d}^{2}+\sigma^{2}}, \quad \zeta=\sigma / \omega_{n}
$$

These relations assume linear damping. However, if the strings are long enough so that the damping ratio is much less than unity, the nonlinearities in the damping will have no significant effect on the frequency. Thus, the mass moment of inertia can be accurately evaluated from the experimentally-determined natural frequency. The data can be fit to a damped sinusoid and from Eqs. (97) and (98) we can use the relation

$$
I_{y y}=\frac{W}{\omega_{d}^{2}+\sigma^{2}}\left(\frac{r_{1}^{2} C_{1}}{s_{1}}+\frac{r_{2}^{2} C_{2}}{s_{2}}+\frac{r_{3}^{2} C_{3}}{s_{3}}\right)
$$

and the radius of gyration can be obtained from

$$
r_{y y} \equiv \sqrt{\frac{g I_{y y}}{W}}=\sqrt{\frac{g}{\omega_{d}^{2}+\sigma^{2}}\left(\frac{r_{1}^{2} C_{1}}{s_{1}}+\frac{r_{2}^{2} C_{2}}{s_{2}}+\frac{r_{3}^{2} C_{3}}{s_{3}}\right)}
$$

To determine the moment of inertia and radius of gyration for pitch, an aircraft is suspended so that the pitch axis is vertical as shown in Fig. 9. The moments of inertia and radii of gyration for roll and yaw can be obtained in a similar manner, by suspending the aircraft in different orientations. This method is not limited to small UAVs. One need only use stronger strings or cables to support larger objects. Variations of this method have long been used to obtain moments of inertia for objects as large as full-scale manned aircraft. ${ }^{41}$ To account for the mass of the strings or cables and any additional structure used to support the object, one can perform the experiment both with and without the object in place, or with an object of known moment of inertia. From the two separate results it is simple to compute the mass properties of the object without the strings or cables and supporting structure. Following a development similar to that presented is Sec. V, it is easily shown that,

$$
\bar{x}_{\text {object }}=\frac{\bar{x}_{\text {total }} W_{\text {total }}-\bar{x}_{\text {support }} W_{\text {support }}}{W_{\text {object }}}, \quad \bar{z}_{\text {object }}=\frac{\bar{z}_{\text {total }} W_{\text {total }}-\bar{z}_{\text {support }} W_{\text {support }}}{W_{\text {object }}}
$$

and

$$
\begin{aligned}
\left(I_{y y}\right)_{\text {object }}= & \left(I_{y y}\right)_{\text {total }}-\left(I_{y y}\right)_{\text {support }} \\
& -\left[\left(\bar{x}_{\text {total }}-\bar{x}_{\text {support }}\right)^{2}+\left(\bar{z}_{\text {total }}-\bar{z}_{\text {support }}\right)^{2}\right]\left(W_{\text {total }} / g\right)\left(W_{\text {support }} / W_{\text {object }}\right)
\end{aligned}
$$




\section{Conclusions}

There is nothing completely original in the material that is presented in this paper. The foundational material was presented by Phillips and Niewoehner. ${ }^{27}$ Variations of the procedure proposed for locating the stick-fixed maneuver point of an unmanned aircraft are commonly used for locating the stick-fixed maneuver point of manned aircraft. ${ }^{35-38}$ Furthermore, variations of the filar pendulum have been used to experimentally determine the mass moments of inertia of airplanes for nearly a century. ${ }^{41-47}$

What this paper does provide is a consolidation of the analytical details associated with procedures that can be used to experimentally determine the traditional control anticipation parameter and the recently-proposed dynamic $\operatorname{margin}^{27}$ for an unmanned aircraft. The procedures and required tools are simple enough to be implemented with an $\mathrm{RC}$ aircraft in an educational setting where resources for extensive flight testing are often limited. With these tools, university students throughout the world could begin to collaborate on the collection of flight-test data that would eventually lead to exposing the boundaries of good flying qualities for remotely-piloted aircraft, in much the same way that the U.S. military has exposed the boundaries of good flying qualities for manned aircraft. ${ }^{3,4}$

Technical societies like the AIAA could help to facilitate such university collaboration through the societysponsored student competitions. For example, in student competitions involving unmanned aircraft, the competition rules could require students to submit certain flight-test data as a prerequisite to qualifying an aircraft for the final phase of the competition.

In any case, we should not expect dynamic stability constraints for UAVs to be the same as those for manned aircraft. The development of more realistic stability constraints for unmanned aircraft will contribute significantly to the safe and efficient operation of UAVs in all applications. The boundaries of controllability for both remotelypiloted and auto-piloted unmanned aircraft must be established before UAV technology can reach its full potential. The procedures described and analyzed in this paper could be used to collect a portion of the data needed to expose the boundaries of good flying qualities for unmanned aircraft.

\section{References}

1“Unmanned Aircraft Operations in the National Airspace System," 14 CFR Part 91, Docket FAA-2006-25714, United States Department of Transportation, Federal Aviation Administration, Washington DC, Feb. 2007.

2"Model Aircraft Operating Standards," Advisory Circular, FAA-AC-91-57, United States Department of Transportation, Federal Aviation Administration, Washington DC, June 1981.

3"Short-Period Frequency and Acceleration Sensitivity," Military Specification - Flying Qualities of Piloted Airplanes, MIL-F-8785C, United States Department of Defense, Philadelphia, PA, Nov. 1980, pp. 13-17.

4"Flying Qualities Requirements for the Pitch Axis," Military Standard - Flying Qualities of Piloted Aircraft, MIL-STD-1797A, United States Department of Defense, Washington DC, Jan. 1990, pp. 167-351.

${ }^{5}$ Hodgkinson, J., "Longitudinal Dynamic Handling Qualities Prediction," Aircraft Handling Qualities, AIAA, Reston, VA, 1999, pp. 35-86.

${ }^{6}$ Foster, T. M., and Bowman, W. J., "Dynamic Stability and Handling Qualities of Small Unmanned-AerialVehicles," AIAA-2005-1023, 43 ${ }^{\text {rd }}$ AIAA Aerospace Sciences Meeting and Exhibit, Reno NV, Jan. 2005.

${ }^{7}$ Foster, T. M., "Dynamic Stability and Handling Qualities of Small Unmanned-Aerial-Vehicles," Thesis, Brigham Young University, Provo UT, April 2005.

${ }^{8}$ Norton, F. H., "The Measurement of the Damping in Roll on a JN4h in Flight," NACA Report 167, 1923.

${ }^{9}$ Norton, F. H., "A Study of Longitudinal Dynamic Stability in Flight," NACA Report 170, 1923.

${ }^{10}$ Tischler, M. B., and Remple, R. K., Aircraft and Rotorcraft System Identification: Engineering Methods with Flight Test Examples, AIAA, Reston, VA, 2006.

${ }^{11}$ Jategaonkar, R. V., Flight Vehicle System Identification: A Time Domain Methodology, AIAA, Reston, VA, 2006.

${ }^{12}$ Klein, V., and Morelli, E. A., Aircraft System Identification: Theory and Practice, AIAA, Reston, VA, 2006.

${ }^{13}$ Iliff, K. W., "Parameter Estimation for Flight Vehicles," Journal of Guidance, Control, and Dynamics, Vol. 12, No. 5, 1989, pp. 609-622.

${ }^{14}$ Mehra, R. K., "Identification of Stochastic Linear Dynamic Systems Using Kalman Filter Representation," AIAA Journal, Vol. 9, No. 1, 1971, pp. 28-31.

${ }^{15}$ Mehra, R. K., Stepner, D. E., and Tyler, J. S., "Maximum Likelihood Identification of Aircraft Stability and Control Derivatives," Journal of Aircraft, Vol. 11, No. 2, 1974, pp. 81-89. 
${ }^{16}$ Morelli, E. A., and Klein, V., "Accuracy of Aerodynamic Model Parameters Estimated from Flight Test Data,"
Journal of Guidance, Control, and Dynamics, Vol. 20, No. 1, 1977, pp. 74-80.
${ }^{17}$ Morelli, E. A., "Low-Order Equivalent System Identification for the Tu-144LL Supersonic Transport Aircraft,"
Journal of Guidance, Control, and Dynamics, Vol. 26, No. 2, 2003, pp. 354-362.
${ }^{18}$ Valasek, J., and Chen, W., "Observer/Kalman Filter Identification for Online System Identification of
Aircraft," Journal of Guidance, Control, and Dynamics, Vol. 26, No. 2, 2003, pp. 347-353.
${ }^{19}$ Morelli, E. A., "Real-Time Parameter Estimation in the Frequency Domain," Journal of Guidance, Control,
and Dynamics, Vol. 23, No. 5, 2000, pp. 812-818.
${ }^{20}$ Mehra, R. K., and Prasanth, R. K., "Time-Domain System Identification Methods for Aeromechanical and Aircraft Structural Modeling," Journal of Aircraft, Vol. 41, No. 4, 2004, pp. 721-729.

${ }^{21}$ Shiryayev, O. V., and Slater, J. C., "Aeroelastic System Identification Using the Minimum Model Error Method," Journal of Guidance, Control, and Dynamics, Vol. 29, No. 4, 2006, pp. 936-943.

${ }^{22}$ Kukreja, S. L., and Brenner, M. J., "Nonlinear Aeroelastic System Identification with Application to Experimental Data," Journal of Guidance, Control, and Dynamics, Vol. 29, No. 2, 2006, pp. 374-381.

${ }^{23}$ Klein, V., and Morelli, E. A., "Regression Methods," Aircraft System Identification: Theory and Practice, AIAA, Reston, VA, 2006, pp. 95-138.

${ }^{24}$ Jategaonkar, R. V., "Least Squares Method," Flight Vehicle System Identification: A Time Domain Methodology, AIAA, Reston, VA, 2006, 178-187.

${ }^{25}$ Maine, R. E., and Iliff, K. W., "Application of Parameter Estimation to Aircraft Stability and Control - The Output-Error Approach,” NASA Report 1168, 1986.

${ }^{26}$ Klein, V., and Morelli, E. A., "Maximum Likelihood Methods," Aircraft System Identification: Theory and Practice, AIAA, Reston, VA, 2006, pp. 181-223.

${ }^{27}$ Phillips, W. F., and Niewoehner, R. J., "Characteristic Length and Dynamic Time Scale Associated with Aircraft Pitching Motion," AIAA-2009-0304, 47th AIAA Aerospace Sciences Meeting and Exhibit, Orlando, FL, Jan. 2009.

${ }^{28}$ Etkin, B., and Reid, L. D., "General Equations of Unsteady Motion," Dynamics of Flight: Stability and Control, 3rd ed., Wiley, New York, NY, 1996, pp. 60-92.

${ }^{29}$ Nelson, R. C., "Aircraft Equations of Motion," Flight Stability and Automatic Control, 2nd ed., McGraw-Hill, New York, 1998, pp. 96-130.

${ }^{30}$ Pamadi, B. N., "Equations of Motion and Estimation of Stability Derivatives," Performance, Stability, Dynamics, and Control of Airplanes, 2nd ed., AIAA, Reston, VA, 2004, pp. 321-442.

${ }^{31}$ Phillips, W. F., "Aircraft Equations of Motion,” Mechanics of Flight, Wiley, Hoboken, NJ, 2004, pp. 599-696.

${ }^{32}$ Yechout, T. R., "Aircraft Equations of Motion," Introduction to Aircraft Flight Mechanics, AIAA, Reston, VA, 2003, pp. 145-172.

${ }^{33}$ Phillips, W. F., “Example 9.8.1,” Mechanics of Flight, Wiley, Hoboken, NJ, 2004, pp. 816-819.

${ }^{34}$ Phillips, W. F., and Snyder, D. O., "Modern Adaptation of Prandtl's Classic Lifting-Line Theory," Journal of Aircraft, Vol. 37, No. 4, 2000, pp. 662-670.

35،"Fixed Wing Stability and Control Theory and Flight Test Techniques," U.S. Naval Test Pilot School Flight Test Manual, USNTPS-FTM-No. 103, Naval Air Warfare Center Aircraft Division, Patuxent River, MD, Revised Jan. 1997.

36“Stability and Control Flight Test Techniques," Stability and Control, Vol. II, AFFTC-TIH-77-1, U.S. Air Force Aerospace Research Pilot School, Edwards Air Force Base, CA, Revised Feb. 1977.

${ }^{37}$ Kimberlin, R. D., "Static Longitudinal Stability Flight Test Methods" and "Maneuvering Stability Methods and Data Reduction," Flight Testing of Fixed-Wing Aircraft, AIAA, Reston, VA, 2003, pp. 223-233 and 265-273.

${ }^{38}$ Ward, D. T., Strganac, T. W., and Niewoehner, R., "Longitudinal Stability Tests" and "Longitudinal Maneuverability Tests," Introduction to Flight Test Engineering Volume One, 3rd ed., Kendall/Hunt Publishing Co., Dubuque, IA, 2006.

${ }^{39}$ Solies, U. P., "Effects of Thrust Line Offset on Neutral Point Determination in Flight Testing," Journal of Aircraft, Vol. 31, No. 2, 1994, pp. 362-366.

${ }^{40}$ Phillips, W. F., and Santana, B. W., "Aircraft Small-Disturbance Theory with Longitudinal-Lateral Coupling," Journal of Aircraft, Vol. 39, No. 6, 2002, pp. 973-980.

${ }^{41}$ Green, M. W., "Measurement of the Moments of Inertia of Full Scale Airplanes," NACA TN-265, 1927.

${ }^{42}$ Miller, M. P., "An Accurate Method of Measuring the Moments of Inertia of Airplanes," NACA TN-351, 1930 . 
${ }^{43}$ Soulé, H. A., and Miller, M. P., "The Experimental Determination of the Moments of Inertia of Airplanes," NACA TR-467, 1934.

${ }^{44}$ Gracey, W., "The Experimental Determination of the Moments of Inertia of Airplanes by a Simplified Compound-Pendulum Method," NACA TN-1629, 1948.

${ }^{45}$ Turner, H. L., "Measurement of the Moments of Inertia of an Airplane by a Simplified Method," NACA TN2201, 1950.

${ }^{46}$ de Jong, R. C., and Mulder, J. A., "Accurate Estimation of Aircraft Inertia Characteristics from a Single Suspension Experiment," Journal of Aircraft, Vol. 24, No. 6, 1987, pp. 362-370.

${ }^{47} J a r d i n$, M. R., and Mueller, E. R., "Optimized Measurements of UAV Mass Moment of Inertia with a Bifilar Pendulum," AIAA-2007-6822, AIAA Guidance, Navigation and Control Conference, Hilton Head SC, Aug. 2007.

${ }^{48}$ Kane, T. R., and Tseng, G.-T., "Dynamics of the Bifilar Pendulum," International Journal of Mechanical Sciences, Vol. 9, Feb., 1967, pp. 83-96.

${ }^{49}$ Lyons, D., "Obtaining Optimal Results with Filar Pendulums for Moment of Inertia Measurements," Paper 3237, Weight Engineering, Society of Allied Weight Engineers, Vol. 62, No. 2, 2002, pp. 5-20. 